\title{
Chemical complexity induced by efficient ice evaporation in the Barnard 5 molecular cloud
}

\author{
V. Taquet ${ }^{1}$, E. S. Wirström ${ }^{2}$, S. B. Charnley ${ }^{3}$, A. Faure ${ }^{4,5}$, A. López-Sepulcre ${ }^{6,7}$, and C. M. Persson ${ }^{2}$ \\ ${ }^{1}$ Leiden Observatory, Leiden University, PO Box 9513, 2300-RA Leiden, The Netherlands \\ e-mail: taquet@strw.leidenuniv.nl \\ 2 Department of Earth and Space Sciences, Chalmers University of Technology, Onsala Space Observatory, 43992 Onsala, Sweden \\ 3 Astrochemistry Laboratory, Mailstop 691, NASA Goddard Space Flight Center, 8800 Greenbelt Road, Greenbelt, MD 20770, USA \\ 4 Univ. Grenoble Alpes, IPAG, 38000 Grenoble, France \\ 5 CNRS, IPAG, 38000 Grenoble, France \\ 6 Department of Physics, The University of Tokyo, 7-3-1 Hongo, Bunkyo-ku, 113-0033 Tokyo, Japan \\ 7 Institut de Radioastronomie Millimétrique, 38400 Grenoble, France
}

Received 7 November 2016 / Accepted 6 June 2017

\begin{abstract}
Cold gas-phase water has recently been detected in a cold dark cloud, Barnard 5 located in the Perseus complex, by targeting methanol peaks as signposts for ice mantle evaporation. Observed morphology and abundances of methanol and water are consistent with a transient non-thermal evaporation process only affecting the outermost ice mantle layers, possibly triggering a more complex chemistry. Here we present the detection of the complex organic molecules $(\mathrm{COMs})$ acetaldehyde $\left(\mathrm{CH}_{3} \mathrm{CHO}\right)$ and methyl formate $\left(\mathrm{CH} \mathrm{H}_{3} \mathrm{OCHO}\right)$, as well as formic acid $(\mathrm{HCOOH})$ and ketene $\left(\mathrm{CH}_{2} \mathrm{CO}\right)$, and the tentative detection of di-methyl ether $\left(\mathrm{CH}_{3} \mathrm{OCH}_{3}\right)$ towards the "methanol hotspot" of Barnard 5 located between two dense cores using the single dish OSO $20 \mathrm{~m}$, IRAM $30 \mathrm{~m}$, and NRO $45 \mathrm{~m}$ telescopes. The high energy cis-conformer of formic acid is detected, suggesting that formic acid is mostly formed at the surface of interstellar grains and then evaporated. The detection of multiple transitions for each species allows us to constrain their abundances through LTE and non-LTE methods. All the considered COMs show similar abundances between $\sim 1$ and $\sim 10 \%$ relative to methanol depending on the assumed excitation temperature. The non-detection of glycolaldehyde, an isomer of methyl formate, with a [glycolaldehyde]/[methyl formate] abundance ratio lower than $6 \%$, favours gas phase formation pathways triggered by methanol evaporation. According to their excitation temperatures derived in massive hot cores, formic acid, ketene, and acetaldehyde have been designated as "lukewarm" COMs whereas methyl formate and di-methyl ether were defined as "warm" species. Comparison with previous observations of other types of sources confirms that lukewarm and warm COMs show similar abundances in low-density cold gas whereas the warm COMs tend to be more abundant than the lukewarm species in warm protostellar cores. This abundance evolution suggests either that warm COMs are indeed mostly formed in protostellar environments and/or that lukewarm COMs are efficiently depleted by increased hydrogenation efficiency around protostars.
\end{abstract}

Key words. astrochemistry - ISM: abundances - ISM: clouds - ISM: molecules - molecular processes - stars: formation

\section{Introduction}

It has been known for more than a decade that the early stages of low-mass star formation are rich in interstellar complex organic molecules (COMs, i.e. molecules based on carbon chemistry and with six atoms or more; Herbst \& van Dishoeck 2009). This knowledge came as a result of the detection of several saturated COMs by Cazaux et al. (2003) and Bottinelli et al. (2004a, 2007) towards nearby bright Class 0 protostars with sub-millimeter single-dish telescopes. Subsequent interferometric observations of Class 0 protostars confirmed that the emission of COMs mostly originates from the inner warm regions of protostellar envelopes, the so-called hot corinos, the low-mass counterparts of the massive hot cores (Bottinelli et al. 2004b; Kuan et al. 2004; Jørgensen et al. 2005, 2011; Maury et al. 2014; Taquet et al. 2015).

The current scenario explaining the detection of warm $(T>$ $100 \mathrm{~K})$ COMs surrounding protostars is mostly based on grain surface chemistry followed by "hot core" gas phase chemistry. In this paradigm, cold $(T \sim 10 \mathrm{~K})$ ices, containing the first "parent" organic molecules such as formaldehyde $\mathrm{H}_{2} \mathrm{CO}$ and methanol $\mathrm{CH}_{3} \mathrm{OH}$ and eventually several other COMs (e.g. ethanol $\mathrm{C}_{2} \mathrm{H}_{5} \mathrm{OH}$, or ethylene glycol $\left.\left(\mathrm{CH}_{2} \mathrm{OH}\right)_{2}\right)$, are formed at the surface of interstellar grains in dark clouds by atom additions on cold dust. It has been hypothesised that more complex molecules could also be formed in lukewarm $(30 \mathrm{~K}<T<$ $80 \mathrm{~K}$ ) ices through the recombination of radicals produced by the UV photolysis of the main ice components during the warmup phase in protostellar envelopes. All the ice content is then evaporated into the gas phase when the temperature exceeds 100 K (Garrod \& Herbst 2006; Garrod et al. 2008). Ion-neutral chemistry triggered by the evaporation of ices, in which ammonia plays a key role, could also be important for the formation of abundant COMs, such as methyl formate $\mathrm{CH}_{3} \mathrm{OCHO}$ or dimethyl ether $\mathrm{CH}_{3} \mathrm{OCH}_{3}$ (Taquet et al. 2016).

The detection of cold methanol, ketene $\mathrm{CH}_{2} \mathrm{CO}$, acetaldehyde $\mathrm{CH}_{3} \mathrm{CHO}$, or formic acid $\mathrm{HCOOH}$ observed in dark clouds for several decades (Matthews et al. 1985; Friberg et al. 1988; Irvine et al. 1989, 1990; Ohishi \& Kaifu 1998) can be explained by the cold surface chemistry scenario mentioned previously followed by non-thermal evaporation processes. However, the UVinduced scenario of COM formation on lukewarm $(T \geq 30 \mathrm{~K})$ 
interstellar grains, invoked to produce methyl formate and dimethyl ether, cannot explain the recent first clear detection by Bacmann et al. (2012) of these two species in a cold $(T \sim 10 \mathrm{~K})$ prestellar core, L1689B, shielded from strong UV radiation. Several COMs have also been detected by Öberg et al. (2010) and Cernicharo et al. (2012) towards the core B1-b. However, more recent interferometric observations suggest that the molecular emission should rather come from nearby protostars, hydrostatic core candidates and outflows (see Gerin et al. 2015). More recently, Vastel et al. (2014) and Jiménez-Serra et al. (2016) also detected $\mathrm{CH}_{3} \mathrm{OH}, \mathrm{HCOOH}, \mathrm{CH}_{2} \mathrm{CO}, \mathrm{CH}_{3} \mathrm{CHO}, \mathrm{CH}_{3} \mathrm{OCHO}$, and $\mathrm{CH}_{3} \mathrm{OCH}_{3}$ towards the prestellar core prototype L1544. A non-LTE analysis of the methanol emission towards L1544 suggests that the emission from COMs would mostly originate from the external part of the core, with an intermediate density $n_{\mathrm{H}}=4 \times 10^{4} \mathrm{~cm}^{-3}$ (Vastel et al. 2014). However, subsequent observations of methanol towards eight other prestellar cores by Bacmann \& Faure (2016) suggest that the cold methanol emission could be associated with denser $\left(n_{\mathrm{H}} \geq 10^{5} \mathrm{~cm}^{-3}\right)$ gas.

New chemical pathways have been proposed to explain the detection of COMs towards these cold regions. Vasyunin \& Herbst (2013) and Balucani et al. (2015) introduced neutral-neutral gas phase reactions triggered by the non-thermal evaporation of methanol assumed to be mostly formed at the surface of grains whilst Reboussin et al. (2014) investigated the effect of heating by cosmic-rays on the surface formation of COMs. Fedoseev et al. (2015) and Chuang et al. (2016) experimentally showed that surface hydrogenation of $\mathrm{CO}$ at low $(T=$ $15 \mathrm{~K})$ temperatures leads to the detection of several COMs through recombination of radicals whose production is triggered by abstraction reactions of formaldehyde and methanol. However, these experiments tend to produce more glycolaldehyde and ethylene glycol, not yet detected towards dense clouds, than methyl formate whilst di-methyl ether cannot be efficiently produced in their experimental setup.

COMs have been detected towards only a few dark cloud regions so far. In this work, we aim to investigate the level of chemical complexity in the so-called methanol hotspot region of the Barnard 5 molecular cloud, one of the two molecular clouds with L1544 where cold water vapour has been detected with Herschel (Caselli et al. 2010, 2012; Wirstrom et al. 2014), by observing mm-emission from COMs with single-dish telescopes. This region, offset from any infrared sources, shows a high abundance of methanol and water attributed to transient evaporation processes (see Wirstrom et al. 2014). In Sect. 2 we describe our observational campaign focussing on detecting oxygen-bearing COMs. In Sect. 3 we present the spectra and the analysis to derive column densities and abundances. In Sect. 4 we discuss the results.

\section{Observations}

\subsection{The "methanol hotspot" in Barnard 5}

The Barnard 5 (B5) dark cloud is located at the North-East of the Perseus complex (235 pc; Hirota et al. 2008). It contains four known protostars of which the most prominent is the Class I protostar IRS1. SCUBA continuum emission maps at $850 \mu \mathrm{m}$ carried out with the JCMT only revealed dust emission towards known protostars and at about 2-4 arcmin north from IRS 1 (Hatchell et al. 2005) whilst molecular maps of CO, $\mathrm{NH}_{3}$, and other species show several chemically differentiated clumps (S. B. Charnley, in prep.). Methanol emission in B5 displays a particularly interesting distribution since it shows a bright so-called methanol hotspot at about 250 arcsec north-west from IRS 1, a region showing no infrared sources and no detected sub-mm continuum emission (see Fig. 1 in Hatchell et al. 2005; Wirstrom et al. 2014). The subsequent detection of abundant water, with absolute abundances of about $10^{-8}$, with the Herschel Space Observatory by Wirstrom et al. (2014) suggests that efficient non-thermal processes triggering the evaporation of icy methanol and water are at work. The methanol hotspot therefore represents an ideal target to detect cold COMs in dark clouds since they are supposed to be formed from methanol, either at the surface of interstellar grains, or directly in the gas phase after the evaporation of methanol. The Perseus molecular cloud was recently mapped with Herschel as part as the Gould Belt Survey key program (André et al. 2010) using the photometers PACS and SPIRE in five bands between $70 \mu \mathrm{m}$ and $500 \mu \mathrm{m}$. Figure 1 presents the Barnard 5 molecular cloud as seen by Herschel/SPIRE at $250 \mu \mathrm{m}$. The Herschel data has been retrieved from the Herschel Science Archive. The data were calibrated and processed with the Herschel pipeline and are considered as a level-3 data product. Dust emission is compared with the integrated intensity map of the $\mathrm{A}^{+}-\mathrm{CH}_{3} \mathrm{OH} 3_{0}-2_{0}$ transition as observed with the IRAM 30 m telescope (this work). The methanol hotspot is located between the two dense cores East 189 and East 286 (Sadavoy, priv. comm.) revealed by Herschel for the first time, and the methanol emission peaks at the edge of core East 189. East 189 and East 286 have the following properties derived through a fitting of their observed spectral energy distribution: $M=0.5 M_{\odot}, T_{\mathrm{d}}=12 \mathrm{~K}, R=3.6 \times 10^{-2} \mathrm{pc}, n_{\mathrm{H}}=$ $3 \times 10^{4} \mathrm{~cm}^{-3}$, and $M=0.7 M_{\odot}, T_{\mathrm{d}}=9.9 \mathrm{~K}, R=2.7 \times 10^{-2} \mathrm{pc}$, $n_{\mathrm{H}}=1.2 \times 10^{5} \mathrm{~cm}^{-3}$ (Sadavoy 2013).

\subsection{Observational details}

We targeted several COMs in different frequency bands at $3 \mathrm{~mm}$ using the NRO $45 \mathrm{~m}$, IRAM $30 \mathrm{~m}$, and OSO $20 \mathrm{~m}$ telescopes and $\mathrm{CH}_{3} \mathrm{OH}$ in two frequency bands at $2 \mathrm{~mm}$ using the IRAM $30 \mathrm{~m}$ towards the methanol hotspot at RA $=3^{\mathrm{h}} 47^{\mathrm{m}} 32 \mathrm{~s} \cdot 10$, decl $=$ $+32^{\mathrm{d}} 56^{\mathrm{m}} 43$ s. 0 . Table 1 summarises the properties of our observational data.

Observations with the NRO $45 \mathrm{~m}$ telescope were carried out in February 2015. We used the TZ1V and TZ1H receivers, connected to the SAM45 spectrometer, tuned to a frequency of $94 \mathrm{GHz}$. Eight $250 \mathrm{MHz}$ bands between 86.3 and $90.3 \mathrm{GHz}$ and between 98.3 and $102.3 \mathrm{GHz}$ were covered with a spectral resolution of $60 \mathrm{kHz}$, corresponding to a velocity resolution of $\sim 0.2 \mathrm{~km} \mathrm{~s}^{-1}$ at $\sim 90 \mathrm{GHz}$. The observations were performed with the position switching mode. Pointing was checked every $1-1.5 \mathrm{~h}$ on a nearby bright source and was found to be accurate within $3^{\prime \prime}$. The weather conditions were good, although unstable on one night, and system temperatures were typically 120 $180 \mathrm{~K}$. The size of the telescope beam at the observing frequency is $\sim 18^{\prime \prime}$, and the aperture $\eta_{\mathrm{ap}}$ and main beam $\eta_{\mathrm{mb}}$ efficiencies are 0.30 and 0.40 , respectively. The $45 \mathrm{~m}$ data were reduced using the Jnewstar software. The scans were checked individually, flagged and coadded. A polynomial order of three was then fitted over line-free regions to correct for baseline oscillations.

Deep observations with the IRAM 30 m telescope towards the methanol hotspot were carried out in January 2015 and March 2015. The EMIR E090 receiver was used and connected to the FTS backend with a $50 \mathrm{kHz}$ resolution. Pointing was checked every $1.5 \mathrm{~h}$ on a nearby bright source and was found to be accurate within $3^{\prime \prime}$. Observations were performed with the frequency switching mode, resulting in a rms of 3-4 mK per $50 \mathrm{kHz}$ spectral channel. The weather conditions were average 

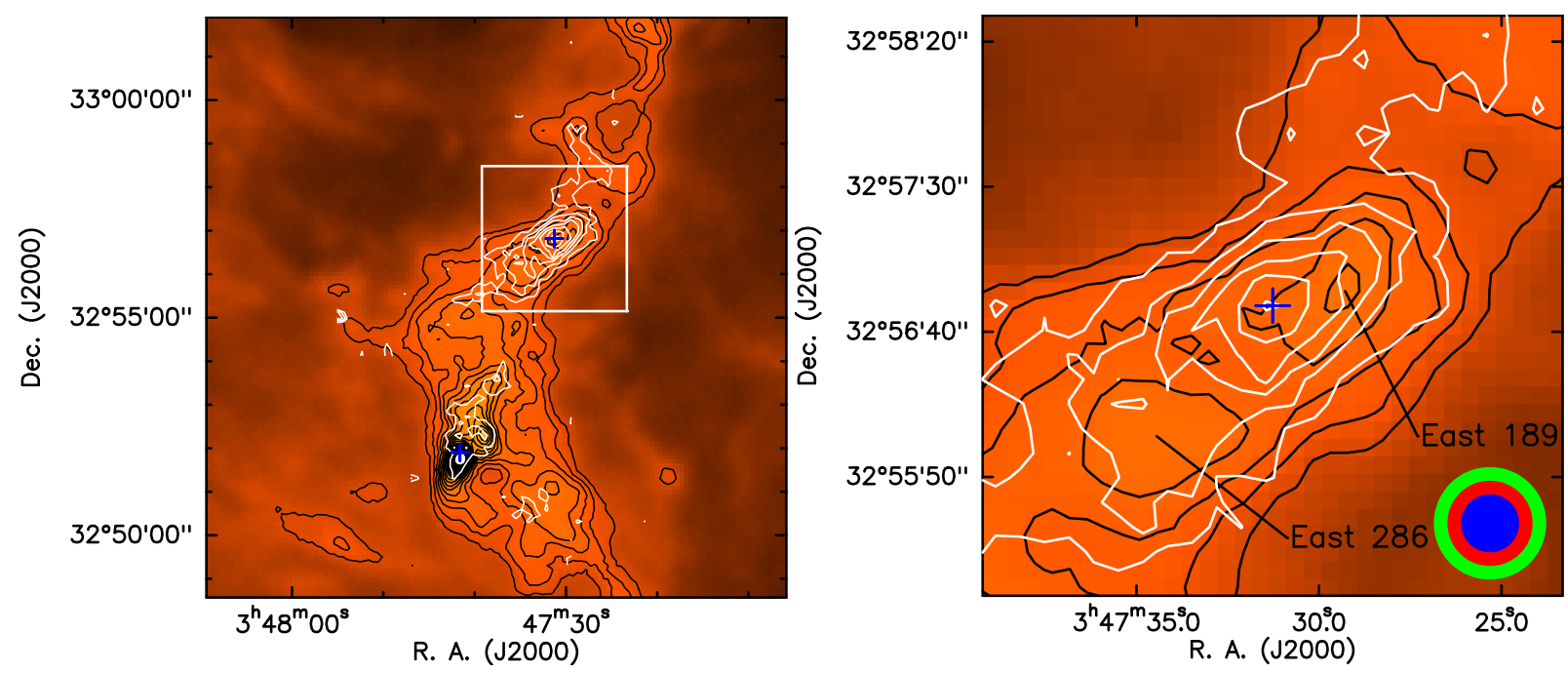

Fig. 1. Herschel/SPIRE map of the Barnard 5 dark cloud at $250 \mu \mathrm{m}$ (orange scale and black contours) and IRAM $30 \mathrm{~m}$ map integrated intensity map of the $\mathrm{A}^{+}-\mathrm{CH}_{3} \mathrm{OH} 3_{0}-2_{0}$ transition at $145.103 \mathrm{GHz}$ (white contours in step of $5 \sigma, \sigma$ is equal to $50 \mathrm{mK} \mathrm{km} \mathrm{s}{ }^{-1}$ ). The blue crosses depict the positions of the Class I protostar IRS1 and the methanol hotspot of B5. The green, red, and blue circles at the bottom right of the right map represent the size of the beams of the OSO $20 \mathrm{~m}$, the IRAM $30 \mathrm{~m}$, and the NRO $45 \mathrm{~m}$ telescopes at $3 \mathrm{~mm}$, respectively. The two dense cores surrounding the methanol hotspot have the following properties: East $189: M=0.5 M_{\odot}, T_{\mathrm{d}}=12 \mathrm{~K}, R=3.6 \times 10^{-2} \mathrm{pc}, n_{\mathrm{H}}=3 \times 10^{4} \mathrm{~cm}^{-3}$, East 286: $M=0.7 M_{\odot}, T_{\mathrm{d}}=9.9 \mathrm{~K}, R=2.7 \times 10^{-2} \mathrm{pc}, n_{\mathrm{H}}=1.2 \times 10^{5} \mathrm{~cm}^{-3}$.

Table 1. Properties of the observations carried out in this work.

\begin{tabular}{|c|c|c|c|c|c|c|c|}
\hline $\begin{array}{l}\text { Frequency range } \\
(\mathrm{GHz})\end{array}$ & Telescope & $\begin{array}{l}\text { Beam size } \\
(\operatorname{arcsec})\end{array}$ & $\begin{array}{c}\mathrm{df} \\
(\mathrm{kHz})\end{array}$ & $\begin{array}{l}\mathrm{rms} \\
(\mathrm{mK})\end{array}$ & $\begin{array}{l}F_{\text {eff }} \\
(\%)\end{array}$ & $\begin{array}{l}B_{\text {eff }} \\
(\%)\end{array}$ & Targeted molecules \\
\hline $83.550-85.370$ & IRAM $30 \mathrm{~m}$ & 29 & 50 & $3-4$ & 95 & 81 & $\mathrm{CH}_{3} \mathrm{OH}, \mathrm{CH}_{3} \mathrm{OCHO}, \mathrm{CH}_{3} \mathrm{OCH}_{3}, \mathrm{CH}_{3} \mathrm{CHO}$ \\
\hline $86.830-88.650$ & IRAM $30 \mathrm{~m}$ & 29 & 50 & $3-4$ & 95 & 81 & $\mathrm{HNCO}, \mathrm{c}-\mathrm{HCOOH}, \mathrm{CH}_{3} \mathrm{OCHO}, \mathrm{CH}_{3} \mathrm{OCH}_{3}$ \\
\hline $89.532-89.629$ & OSO $20 \mathrm{~m}$ & 41 & 60 & 8 & - & 49 & $\mathrm{t}-\mathrm{HCOOH}$ \\
\hline $89.190-89.370$ & $\mathrm{NRO} 45 \mathrm{~m}$ & 20 & 61 & $3-5$ & - & 44 & $\mathrm{CH}_{3} \mathrm{OCHO}$ \\
\hline $89.350-89.530$ & $\mathrm{NRO} 45 \mathrm{~m}$ & 20 & 61 & $5-6$ & - & 44 & $\mathrm{CH}_{2} \mathrm{DOH}$ \\
\hline $89.530-89.780$ & $\mathrm{NRO} 45 \mathrm{~m}$ & 20 & 61 & $4-5$ & - & 44 & $\mathrm{CH}_{3} \mathrm{OCH}_{3}$ \\
\hline $90.075-90.325$ & $\mathrm{NRO} 45 \mathrm{~m}$ & 20 & 61 & $5-6$ & - & 44 & $\mathrm{CH}_{3} \mathrm{OCHO}$ \\
\hline $95.901-96.001$ & OSO $20 \mathrm{~m}$ & 38 & 64 & $6-7$ & - & 50 & $\mathrm{E}-\mathrm{CH}_{3} \mathrm{CHO}, \mathrm{A}-\mathrm{CH}_{3} \mathrm{CHO}$ \\
\hline $96.020-97.840$ & IRAM $30 \mathrm{~m}$ & 25 & 50 & $27-30$ & 94 & 80 & $\mathrm{CH}_{3} \mathrm{OH}$ \\
\hline $98.740-98.990$ & NRO $45 \mathrm{~m}$ & 18 & 61 & $4-5$ & - & 42 & $\mathrm{E}-\mathrm{CH}_{3} \mathrm{CHO}, \mathrm{A}-\mathrm{CH}_{3} \mathrm{CHO}$ \\
\hline $99.200-99.450$ & $\mathrm{NRO} 45 \mathrm{~m}$ & 18 & 61 & $4-5$ & - & 42 & $\mathrm{CH}_{3} \mathrm{OCH}_{3}$ \\
\hline $99.230-101.050$ & IRAM $30 \mathrm{~m}$ & 25 & 50 & $3-4$ & 94 & 80 & $\mathrm{CH}_{3} \mathrm{OCHO}, \mathrm{CH}_{3} \mathrm{OCH}_{3}$ \\
\hline $100.942-101.558$ & OSO 20 m & 36 & 68 & 5 & - & 42 & $\mathrm{p}-\mathrm{CH}_{2} \mathrm{CO}$ \\
\hline $100.910-101.160$ & $\mathrm{NRO} 45 \mathrm{~m}$ & 18 & 61 & $4-5$ & - & 42 & $\mathrm{CH}_{2} \mathrm{CO}, \mathrm{CH}_{3} \mathrm{CHO}$ \\
\hline $101.675-102.290$ & OSO $20 \mathrm{~m}$ & 36 & 68 & 6 & - & 47 & $\mathrm{O}-\mathrm{CH}_{2} \mathrm{CO}$ \\
\hline $102.510-104.330$ & IRAM $30 \mathrm{~m}$ & 25 & 50 & $3-4$ & 94 & 80 & $\mathrm{CH}_{3} \mathrm{OCHO}$ \\
\hline $143.800-145.620$ & IRAM $30 \mathrm{~m}$ & 16 & 50 & $35-40$ & 93 & 73 & $\mathrm{CH}_{3} \mathrm{OH}$ \\
\hline $156.300-158.120$ & IRAM $30 \mathrm{~m}$ & 15 & 50 & $35-40$ & 93 & 73 & $\mathrm{CH}_{3} \mathrm{OH}$ \\
\hline
\end{tabular}

to good, and gave system temperatures of $70-80 \mathrm{~K}$ at $3 \mathrm{~mm}$. A low polynomial order of three or four was then fitted over linefree regions to correct for local baseline oscillations.

On-the-fly observations of $\mathrm{CH}_{3} \mathrm{OH}$ transitions towards the Barnard 5 cloud with the IRAM $30 \mathrm{~m}$ telescope were carried out in February 2017 using the position switching mode with a common centre at IRS1. The EMIR E090 and E150 receivers were used and connected to the FTS backend with a $50 \mathrm{kHz}$ resolution. Pointing was checked every $1.5 \mathrm{~h}$ on a nearby bright source and was found to be accurate within $3^{\prime \prime}$. Position switching observations resulted in a rms of $30-40 \mathrm{mK}$ per $50 \mathrm{kHz}$ spectral channel. The size of the telescope beam is about $25-30^{\prime \prime}$ at $3 \mathrm{~mm}$ and about $\sim 15^{\prime \prime}$ at $2 \mathrm{~mm}$. A low polynomial order of one was then fitted over line-free regions to correct for local baseline oscillations.
The $30 \mathrm{~m}$ data were reduced using the GILDAS/CLASS software. The scans were checked individually, coadded, and eventually folded to deconvolve the spectra from the frequencyswitching procedure. The $1.8 \mathrm{GHz}$ spectral windows covered by our observations are listed in Table 1. Pointing was checked every $1.5 \mathrm{~h}$ on a nearby bright source and was found to be accurate within $3^{\prime \prime}$. A more comprehensive analysis of the $\mathrm{CH}_{3} \mathrm{OH}$ maps in the B5 dark cloud will be presented in a separate publication.

Observations with the Onsala $20 \mathrm{~m}$ telescope were carried out in May 2014 and March 2015. We used the dual-polarisation, sideband-separating $3 \mathrm{~mm}$ receiver system (Belitsky et al. 2015) to target four different frequency settings: 89.58 and $95.95 \mathrm{GHz}$ in 2014, and 101.25 and 101.98 in 2015. In 2014 the spectra were recorded using a fast Fourier transform spectrometer (FFTS) at channel separation $12.2 \mathrm{kHz}$, covering $100 \mathrm{MHz}$ bandwidth. 


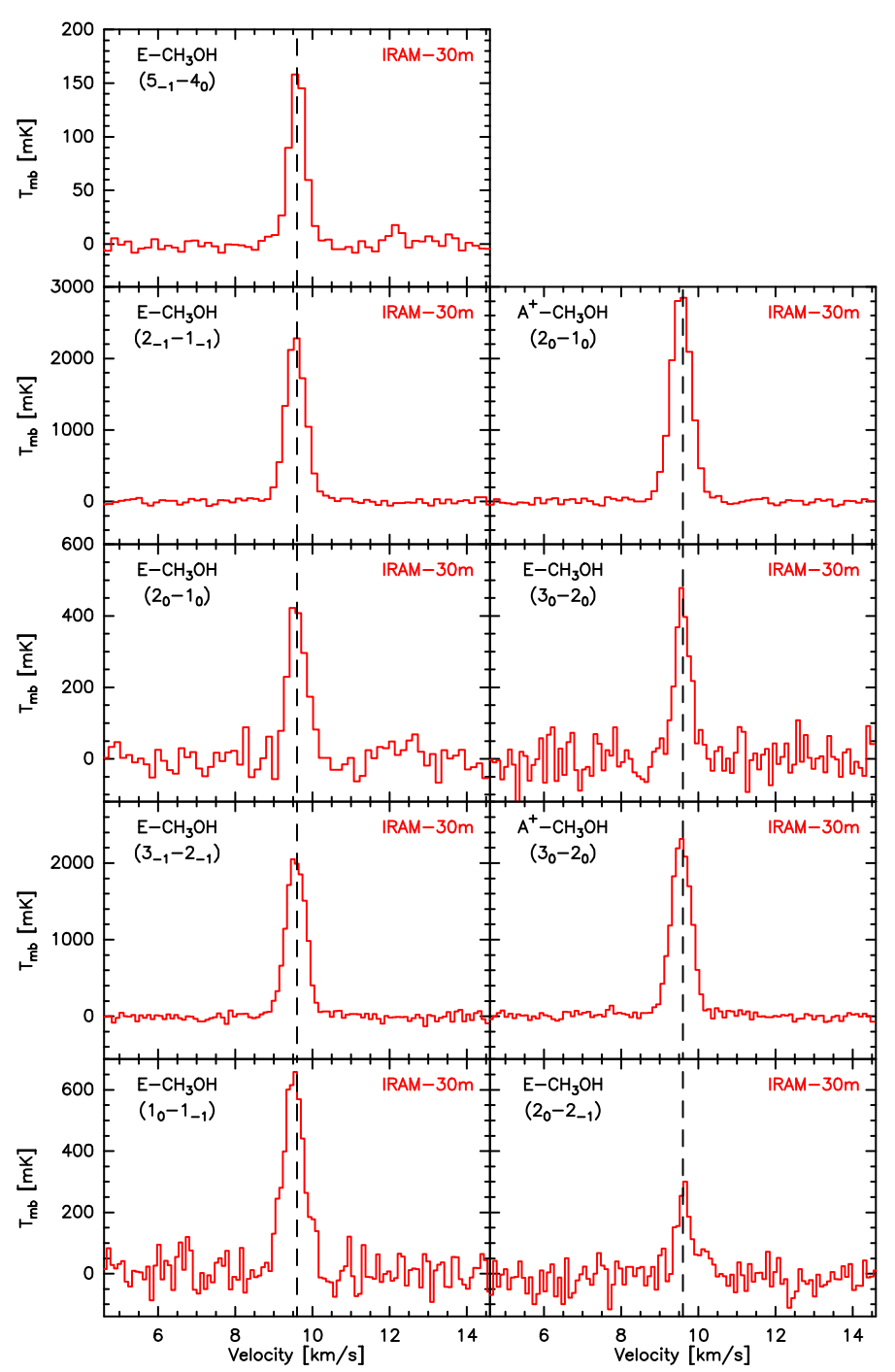

Fig. 2. Spectrum of the targeted $\mathrm{CH}_{3} \mathrm{OH}$ transitions towards the $\mathrm{B} 5$ methanol hotspot.

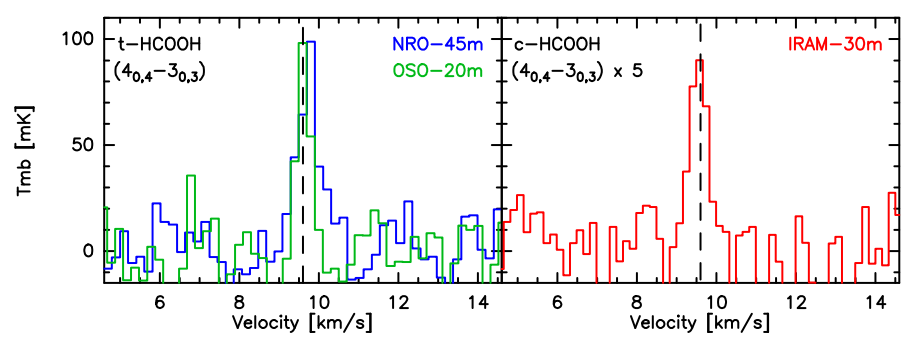

Fig. 3. Spectra of the targeted $\mathrm{HCOOH}$ transitions towards the $\mathrm{B} 5$ methanol hotspot.

Dual beam switching mode was used with an 11' beam throw, and the system temperature varied between 145-235 K. In 2015, a new FFTS of improved bandwidth was used at channel separation $19.1 \mathrm{kHz}$, covering $625 \mathrm{MHz}$ wide spectral windows. These observations were performed in the frequency switching mode with a switching frequency of $5 \mathrm{~Hz}$ and a throw of $3 \mathrm{MHz}$. Conditions were good with system temperatures in the range 160$250 \mathrm{~K}$ and pointing and focus were checked towards strong continuum sources after sunrise and sunset for all observations. The OSO20 m beam full width at half maximum (FWHM) is about $41^{\prime \prime}$ at $89 \mathrm{GHz}, 38^{\prime \prime}$ at $95 \mathrm{GHz}$ and $36^{\prime \prime}$ at $101 \mathrm{GHz}$, and the main

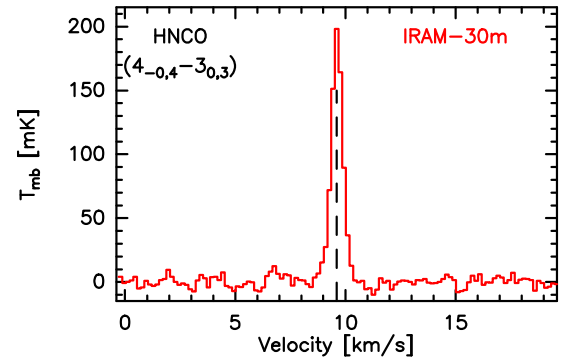

Fig. 4. Spectrum of the targeted HNCO transition at $89.579 \mathrm{GHz}$ towards the B5 methanol hotspot.

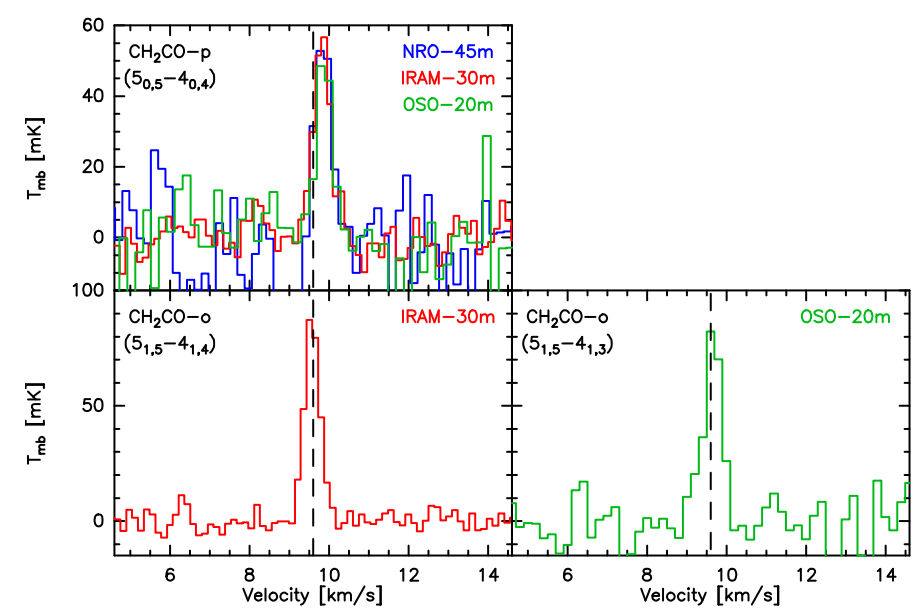

Fig. 5. Spectra of the targeted $\mathrm{CH}_{2} \mathrm{CO}$ transitions at $\sim 101 \mathrm{GHz}$ towards the B5 methanol hotspot.

beam efficiency varies with both frequency and source elevation, resulting in values $0.42-0.50$ for the current observations. The $20 \mathrm{~m}$ data were checked and reduced using the spectral analysis software $\mathrm{XS}^{1}$. Linear baselines were fitted to line-free regions and subtracted from individual, dual polarisation spectra before averaging them together, weighted by rms noise. For the frequency switched spectra, which tend to exhibit low-intensity standing wave features, an additional baseline of the order of between three and five was fitted locally around each line and subtracted before further analysis.

\section{Results}

\subsection{Spectra}

Figures 2-8 present the spectra obtained with the NRO $45 \mathrm{~m}$, the IRAM $30 \mathrm{~m}$, and the OSO $20 \mathrm{~m}$ telescopes. Table 2 presents the frequencies, the spectroscopic parameters, and the observed properties of the targeted transitions. The rms achieved in the observations ranges between 3 and $8 \mathrm{mK}$ per $50-60 \mathrm{kHz}$ spectral bin for deep observations of COMs and between $27-40 \mathrm{mK}$ for observations of bright methanol transitions, depending on the observed frequency and the used telescope. We report here the detection of several transitions from methanol $\left(\mathrm{CH}_{3} \mathrm{OH}\right)$, formic acid $(\mathrm{HCOOH})$, ketene $\left(\mathrm{CH}_{2} \mathrm{CO}\right)$, acetaldehyde $\left(\mathrm{CH}_{3} \mathrm{CHO}\right)$, methyl formate $\left(\mathrm{CH}_{3} \mathrm{OCHO}\right)$, and the tentative detection of dimethyl ether $\left(\mathrm{CH}_{3} \mathrm{OCH}_{3}\right)$.

1 Developed by Per Bergman at Onsala Space Observatory, Sweden; http://www. chalmers.se/rss/oso-en/observations/ data-reduction-software 


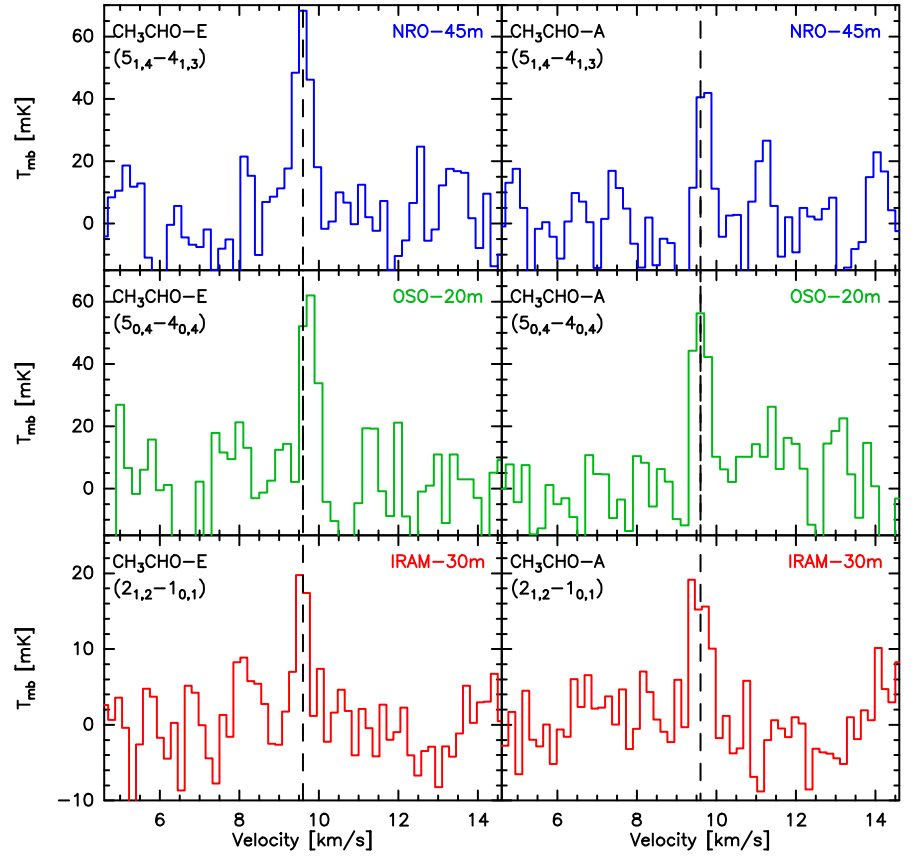

Fig. 6. Spectra of the targeted $\mathrm{CH}_{3} \mathrm{CHO}$ transitions towards the $\mathrm{B} 5$ methanol hotspot.
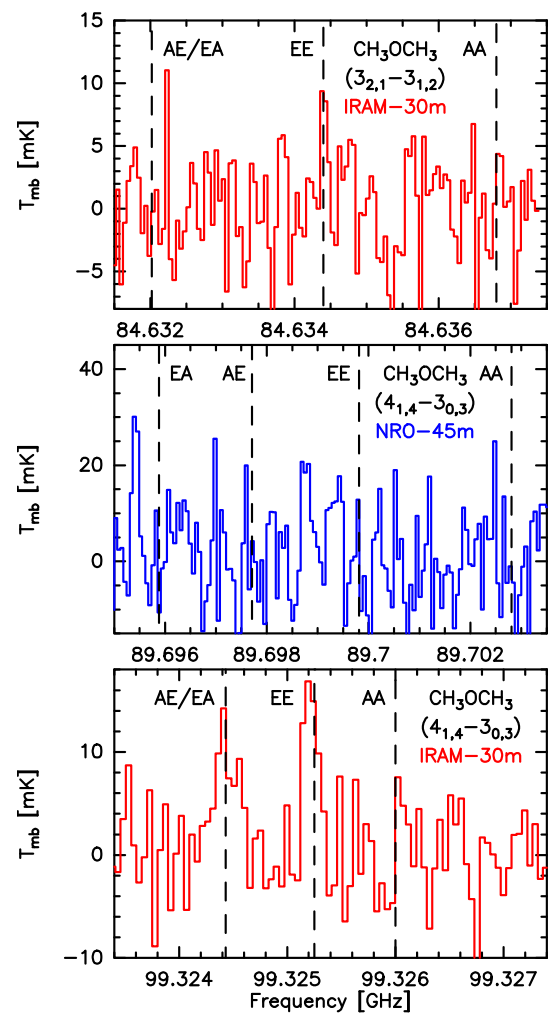

Fig. 7. Spectra of the targeted $\mathrm{CH}_{3} \mathrm{OCH}_{3}$ transitions towards the $\mathrm{B} 5$ methanol hotspot.

We detected the $4_{0,4}-3_{0,3}$ transition of $\mathrm{HCOOH}$ in its two conformers trans and cis at 89.579 and $87.695 \mathrm{GHz}$, respectively (Fig. 3). To our knowledge, this is the second published detection of the higher energy cis- $\mathrm{HCOOH}$ conformer in space after the detection by Cuadrado et al. (2016) towards the Orion bar. Three transitions from ketene, two in its ortho substate and one in its para substate, have been detected (Fig. 5). The para transition of ketene at $101.036 \mathrm{GHz}$ has been detected with all telescopes. The integrated main-beam temperatures obtained with the three telescopes are very similar, the differences in the peak main-beam temperatures remaining within the uncertainties. This comparison suggests that the size of the formic acid and ketene emissions is much larger than 40 arcsec, the beam of the OSO $20 \mathrm{~m}$ at $90 \mathrm{GHz}$. In the following, we therefore assume that the emission size is much larger than the telescope beams for all the considered species, resulting in a beam dilution of one.

Six acetaldehyde transitions, three for each of the substates $\mathrm{A}$ and $\mathrm{E}$, have also been detected with upper level energies between 5 and $17 \mathrm{~K}$ (Fig. 6). $\mathrm{CH}_{3} \mathrm{OCH}_{3}$ is detected in the four substates $\mathrm{AA}, \mathrm{EE}, \mathrm{AE}$, and $\mathrm{EA}$ of the $4_{1,4}-3_{0,3}$ transition whilst the $3_{2,1}-3_{1,2}$ transition at $84 \mathrm{GHz}$ is detected for substate EE only (Fig. 7). The non-detection of the transitions from the other states is in good agreement with observations of other di-methyl ether transitions suggesting that the EE substate usually shows the brightest transition (see Bacmann et al. 2012). Among the 20 transitions from methyl formate targetted with the IRAM $30 \mathrm{~m}$ and the NRO $45 \mathrm{~m}$ telescopes, 13 transitions have been detected at $3 \sigma$ level, five for the E substate and eight in the A substate (Fig. 8). The non-detection of seven transitions, with similar properties, is in good agreement with the high uncertainties of the observed transitions, detected at a signal-to-noise ratio slightly higher than three and showing large uncertainties.

The full width at half maximum (FWHM) of the detected transitions shows a large variation between 0.3 and $0.7 \mathrm{~km} \mathrm{~s}^{-1}$, especially for the faint transitions with a low signal-to-noise ratio. Brighter transitions from methanol or ketene show a FWHM linewidth between 0.5 and $0.6 \mathrm{~km} \mathrm{~s}^{-1}$, as expected for transitions originated from cold dark clouds. Linewidths in B5 tend to be slightly larger than the linewidth of $0.4 \mathrm{~km} \mathrm{~s}^{-1}$ for the transitions of COMs detected by Bacmann et al. (2012) towards L1689B and Jiménez-Serra et al. (2016) towards L1544. For non-detected transitions, we defined a $3 \sigma$ upper limit as $3 \sigma \sqrt{F W H M \times \Delta v}$ where $\sigma$ is the rms noise, FWHM is the typical linewidth of the transition, and $\Delta v$ is the spectral velocity resolution.

\subsection{Non-LTE analysis}

Cross-sections for the rotational excitation of the A- and E-types of methanol and methyl formate by $\mathrm{H}_{2}$ and helium, respectively, and of $\mathrm{HNCO}$ by $\mathrm{H}_{2}$ have been computed by Rabli \& Flower (2010), Faure et al. (2014), and Green (1986), allowing us to perform a non-LTE analysis of the emission of these molecules. To this end, we used the non-LTE radiative transfer code RADEX assuming an isothermal and homogeneous medium using the large velocity approximation for a uniform sphere (van der Tak et al. 2007). The line excitation temperatures $T_{\mathrm{ex}}$, the opacities $\tau$, and the integrated brightness temperatures $T_{\mathrm{mb}}$ of methanol have first been computed by varying the column density of each sub-type $N_{\text {tot }}$ between $10^{13}$ and $10^{15} \mathrm{~cm}^{-2}$ with 20 logarithmic steps, the density of $\mathrm{H}_{2} n_{\mathrm{H} 2}$ between $10^{4}$ and $10^{6} \mathrm{~cm}^{-3}$ with 20 logarithmic steps, and the kinetic temperature $T_{\text {kin }}$ between 5 and $15 \mathrm{~K}$ with 10 linear steps. Due to the low number of detected A- $\mathrm{CH}_{3} \mathrm{OH}$ transitions, we assumed a state $\mathrm{E} / \mathrm{A}$ abundance ratio of one following the observations by Bacmann \& Faure (2016) towards a sample of prestellar cores. The FWHM linewidth was fixed to $0.5 \mathrm{~km} \mathrm{~s}^{-1}$ following our observed spectra. Figure 9 presents the reduced $\chi^{2}$ distribution in 
Table 2. Targeted transitions with their spectroscopic and observed properties.

\begin{tabular}{|c|c|c|c|c|c|c|c|c|}
\hline Molecule & Transition & $\begin{array}{c}\text { Frequency } \\
(\mathrm{GHz})\end{array}$ & $\begin{array}{l}E_{\text {up }} \\
(\mathrm{K})\end{array}$ & $\begin{array}{c}A_{\mathrm{i}, \mathrm{j}} \\
\left(\mathrm{s}^{-1}\right)\end{array}$ & Telescope & $\begin{array}{c}\int_{\left(\mathrm{mK} \mathrm{km} \mathrm{s}^{-1}\right)} T_{\mathrm{mb}}^{*} \mathrm{~d} v \\
\end{array}$ & $\begin{array}{c}\text { Vpeak } \\
\left(\mathrm{km} \mathrm{s}^{-1}\right)\end{array}$ & $\begin{array}{l}F W H M \\
\left(\mathrm{~km} \mathrm{~s}^{-1}\right)\end{array}$ \\
\hline $\mathrm{E}-\mathrm{CH}_{3} \mathrm{OH}$ & $5_{-1}-4_{0}$ & 84.52117 & 40.4 & $2.0 \times 10^{-6}$ & IRAM-30 m & $88.8 \pm 17.9$ & 9.62 & 0.50 \\
\hline $\mathrm{E}-\mathrm{CH}_{3} \mathrm{OH}$ & $2_{-1}-1_{-1}$ & 96.73936 & 12.5 & $2.6 \times 10^{-6}$ & IRAM-30 m & $1490 \pm 300$ & 9.57 & 0.61 \\
\hline $\mathrm{A}^{+}-\mathrm{CH}_{3} \mathrm{OH}$ & $2_{0}-1_{0}$ & 96.74137 & 7.0 & $3.4 \times 10^{-6}$ & IRAM-30 m & $1950 \pm 390$ & 9.56 & 0.62 \\
\hline $\mathrm{E}-\mathrm{CH}_{3} \mathrm{OH}$ & $2{ }_{0}-1_{0}$ & 96.74454 & 20.1 & $3.4 \times 10^{-6}$ & IRAM-30 m & $256 \pm 53$ & 9.60 & 0.55 \\
\hline $\mathrm{E}-\mathrm{CH}_{3} \mathrm{OH}$ & $3_{0}-2_{0}$ & 145.09375 & 27.1 & $1.2 \times 10^{-5}$ & IRAM-30 m & $209 \pm 44$ & 9.59 & 0.47 \\
\hline $\mathrm{E}-\mathrm{CH}_{3} \mathrm{OH}$ & $3-1-2-1$ & 145.09744 & 19.5 & $1.1 \times 10^{-5}$ & IRAM-30 m & $1250 \pm 250$ & 9.57 & 0.60 \\
\hline $\mathrm{A}^{+}-\mathrm{CH}_{3} \mathrm{OH}$ & $3_{0}-2_{0}$ & 145.10319 & 13.9 & $1.2 \times 10^{-5}$ & IRAM-30 m & $1420 \pm 284$ & 9.56 & 0.61 \\
\hline $\mathrm{E}-\mathrm{CH}_{3} \mathrm{OH}$ & $1_{0}-1_{-1}$ & 157.27083 & 15.4 & $2.2 \times 10^{-5}$ & IRAM-30 m & $410 \pm 84$ & 9.52 & 0.65 \\
\hline $\mathrm{E}-\mathrm{CH}_{3} \mathrm{OH}$ & $2_{0}-2_{-1}$ & 157.27606 & 20.1 & $2.2 \times 10^{-5}$ & IRAM-30 m & $114 \pm 27$ & 9.63 & 0.43 \\
\hline $\mathrm{HNCO}$ & $4_{0,4}-3_{0,3}$ & 87.92525 & 10.5 & $8.5 \times 10^{-6}$ & IRAM-30 m & $126 \pm 25$ & 9.63 & 0.61 \\
\hline \multirow[t]{2}{*}{$\mathrm{t}-\mathrm{HCOOH}$} & $4_{0,4}-3_{0,3}$ & 89.57917 & 10.8 & $7.2 \times 10^{-6}$ & NRO-45 m & $56.8 \pm 15.9$ & 9.74 & 0.68 \\
\hline & & & & & OSO-20 m & $39.2 \pm 6.5$ & 9.70 & 0.39 \\
\hline c-HCOOH & $4_{0,4}-3_{0,3}$ & 87.69469 & 10.5 & $2.5 \times 10^{-5}$ & IRAM-30 m & $11.4 \pm 3.3$ & 9.50 & 0.56 \\
\hline $\mathrm{o}-\mathrm{CH}_{2} \mathrm{CO}$ & $5_{1,5}-4_{1,4}$ & 100.09451 & 27.5 & $1.0 \times 10^{-5}$ & IRAM-30 m & $45.8 \pm 9.2$ & 9.58 & 0.61 \\
\hline \multirow[t]{3}{*}{$\mathrm{p}-\mathrm{CH}_{2} \mathrm{CO}$} & $5_{0,5}-4_{0,4}$ & 101.03671 & 14.5 & $1.1 \times 10^{-5}$ & NRO-45 m & $29.5 \pm 8.8$ & 9.85 & 0.50 \\
\hline & & & & & IRAM-30 m & $31.4 \pm 6.4$ & 9.83 & 0.52 \\
\hline & & & & & OSO-20 m & $25.6 \pm 6.5$ & 9.88 & 0.46 \\
\hline $\mathrm{o}-\mathrm{CH}_{2} \mathrm{CO}$ & $5_{1,4}-4_{1,3}$ & 101.98140 & 27.7 & $1.1 \times 10^{-5}$ & OSO-20 m & $46.8 \pm 11.1$ & 9.66 & 0.52 \\
\hline $\mathrm{E}-\mathrm{CH}_{3} \mathrm{CHO}$ & $2_{1,2}-1_{0,1}$ & 83.58426 & 5.03 & $2.4 \times 10^{-6}$ & IRAM-30 m & $8.4 \pm 2.1$ & 9.57 & 0.36 \\
\hline $\mathrm{A}-\mathrm{CH}_{3} \mathrm{CHO}$ & $2_{1,2}-1_{0,1}$ & 84.21976 & 4.96 & $2.4 \times 10^{-6}$ & IRAM-30 m & $11.4 \pm 3.3$ & 9.57 & 0.56 \\
\hline $\mathrm{E}-\mathrm{CH}_{3} \mathrm{CHO}$ & $5_{0,5}-4_{0,4}$ & 95.94744 & 13.9 & $3.0 \times 10^{-5}$ & OSO-20 m & $31.4 \pm 8.7$ & 9.76 & 0.43 \\
\hline $\mathrm{A}-\mathrm{CH}_{3} \mathrm{CHO}$ & $5_{0,5}-4_{0,4}$ & 95.96346 & 13.8 & $3.0 \times 10^{-5}$ & OSO-20 m & $30.4 \pm 8.5$ & 9.62 & 0.46 \\
\hline $\mathrm{E}-\mathrm{CH}_{3} \mathrm{CHO}$ & $5_{1,4}-4_{1,3}$ & 98.86331 & 16.6 & $3.0 \times 10^{-5}$ & NRO-45 m & $20.0 \pm 6.9$ & 9.70 & 0.37 \\
\hline $\mathrm{A}-\mathrm{CH}_{3} \mathrm{CHO}$ & $5_{1,4}-4_{1,3}$ & 98.90094 & 16.5 & $3.0 \times 10^{-5}$ & NRO-45 m & $38.1 \pm 9.5$ & 9.59 & 0.54 \\
\hline $\mathrm{AE} / \mathrm{EA}-\mathrm{CH}_{3} \mathrm{OCH}_{3}$ & $3_{2,1}-3_{1,2}$ & 84.63202 & 11.1 & $2.2 \times 10^{-6}$ & IRAM-30 m & $<3.5$ & & \\
\hline $\mathrm{EE}-\mathrm{CH}_{3} \mathrm{OCH}_{3}$ & $3_{2,1}-3_{1,2}$ & 84.63440 & 11.1 & $2.2 \times 10^{-6}$ & IRAM-30 m & $4.0 \pm 1.4$ & 9.56 & 0.34 \\
\hline $\mathrm{AA}-\mathrm{CH}_{3} \mathrm{OCH}_{3}$ & $3_{2,1}-3_{1,2}$ & 84.6368 & 11.1 & $2.2 \times 10^{-6}$ & IRAM-30 m & $<3.5$ & & \\
\hline $\mathrm{EA}-\mathrm{CH}_{3} \mathrm{OCH}_{3}$ & $2_{2,1}-2_{1,2}$ & 89.69588 & 8.4 & $1.9 \times 10^{-6}$ & NRO-45 m & $<9.4$ & & \\
\hline $\mathrm{AE}-\mathrm{CH}_{3} \mathrm{OCH}_{3}$ & $2_{2,1}-2_{1,2}$ & 89.69771 & 8.4 & $1.9 \times 10^{-6}$ & NRO-45 m & $<9.4$ & & \\
\hline $\mathrm{EE}-\mathrm{CH}_{3} \mathrm{OCH}_{3}$ & $2_{2,1}-2_{1,2}$ & 89.69981 & 8.4 & $1.9 \times 10^{-6}$ & NRO-45 m & $<9.4$ & & \\
\hline $\mathrm{AA}-\mathrm{CH}_{3} \mathrm{OCH}_{3}$ & $2_{2,1}-2_{1,2}$ & 89.70281 & 8.4 & $1.9 \times 10^{-6}$ & NRO-45 m & $<9.4$ & & \\
\hline $\mathrm{AE} / \mathrm{EA}-\mathrm{CH}_{3} \mathrm{OCH}_{3}$ & $4_{1,4}-3_{0,3}$ & 99.32443 & 10.2 & $4.4 \times 10^{-6}$ & IRAM-30 m & $3.9 \pm 2.5$ & 9.57 & 0.39 \\
\hline $\mathrm{EE}-\mathrm{CH}_{3} \mathrm{OCH}_{3}$ & $4_{1,4}-3_{0,3}$ & 99.32525 & 10.2 & $4.4 \times 10^{-6}$ & IRAM-30 m & $9.2 \pm 2.3$ & 9.69 & 0.46 \\
\hline $\mathrm{AA}-\mathrm{CH}_{3} \mathrm{OCH}_{3}$ & $4_{1,4}-3_{0,3}$ & 99.32600 & 10.2 & $4.4 \times 10^{-6}$ & IRAM-30 m & $2.8 \pm 1.3$ & 9.41 & 0.27 \\
\hline $\mathrm{E}-\mathrm{CH}_{3} \mathrm{OCHO}$ & $7_{2,6}-6_{2,5}$ & 84.44917 & 19.0 & $8.0 \times 10^{-6}$ & IRAM-30 m & $11.4 \pm 3.3$ & 9.38 & 0.65 \\
\hline $\mathrm{A}-\mathrm{CH}_{3} \mathrm{OCHO}$ & $7_{2,6}-6_{2,5}$ & 84.45475 & 19.0 & $8.0 \times 10^{-6}$ & IRAM-30 m & $13.1 \pm 3.5$ & 9.70 & 0.47 \\
\hline $\mathrm{E}-\mathrm{CH}_{3} \mathrm{OCHO}$ & $73,4-6,3$ & 87.14328 & 22.6 & $7.7 \times 10^{-6}$ & IRAM-30 m & $6.9 \pm 2.7$ & 9.50 & 0.43 \\
\hline $\mathrm{A}-\mathrm{CH}_{3} \mathrm{OCHO}$ & $7_{3,4}-6_{3,3}$ & 87.16129 & 22.6 & $7.8 \times 10^{-6}$ & IRAM-30 m & $4.7 \pm 2.6$ & 9.62 & 0.58 \\
\hline $\mathrm{E}-\mathrm{CH}_{3} \mathrm{OCHO}$ & $8_{1,8}-7_{1,7}$ & 89.31466 & 20.2 & $1.0 \times 10^{-5}$ & NRO-45 m & $<5.9$ & & \\
\hline $\mathrm{A}-\mathrm{CH}_{3} \mathrm{OCHO}$ & $8_{1,8}-7_{1,7}$ & 89.31664 & 20.1 & $1.0 \times 10^{-5}$ & NRO-45 m & $22.5 \pm 7.3$ & 9.67 & 0.72 \\
\hline $\mathrm{E}-\mathrm{CH}_{3} \mathrm{OCHO}$ & $7_{2,5}-6_{2,4}$ & 90.14572 & 19.7 & $9.8 \times 10^{-6}$ & NRO-45 m & $<9.9$ & & \\
\hline $\mathrm{A}-\mathrm{CH}_{3} \mathrm{OCHO}$ & $7_{2,5}-6_{2,4}$ & 90.15647 & 19.7 & $9.8 \times 10^{-6}$ & NRO-45 m & $17.3 \pm 8.2$ & 9.55 & 0.46 \\
\hline $\mathrm{E}-\mathrm{CH}_{3} \mathrm{OCHO}$ & $8_{0,8}-7_{0,7}$ & 90.22766 & 20.1 & $1.1 \times 10^{-5}$ & NRO-45 m & $<9.9$ & & \\
\hline $\mathrm{A}-\mathrm{CH}_{3} \mathrm{OCHO}$ & $8_{0,8}-7_{0,7}$ & 90.22962 & 20.1 & $1.1 \times 10^{-5}$ & NRO-45 m & $<9.9$ & & \\
\hline $\mathrm{E}-\mathrm{CH}_{3} \mathrm{OCHO}$ & $9_{1,9}-8_{1,8}$ & 100.07861 & 24.9 & $1.4 \times 10^{-5}$ & IRAM-30 m & $3.4 \pm 1.4$ & 9.65 & 0.31 \\
\hline $\mathrm{A}-\mathrm{CH}_{3} \mathrm{OCHO}$ & $9_{1,9}-8_{1,8}$ & 100.08054 & 24.9 & $1.5 \times 10^{-5}$ & IRAM-30 m & $6.0 \pm 1.7$ & 9.50 & 0.30 \\
\hline $\mathrm{E}-\mathrm{CH}_{3} \mathrm{OCHO}$ & $8_{3,5}-7_{3,4}$ & 100.29460 & 27.4 & $1.3 \times 10^{-5}$ & IRAM-30 m & $<2.8$ & & \\
\hline $\mathrm{A}-\mathrm{CH}_{3} \mathrm{OCHO}$ & $8_{3,5}-7_{3,4}$ & 100.30818 & 27.4 & $1.3 \times 10^{-5}$ & IRAM-30 m & $<3.1$ & & \\
\hline $\mathrm{E}-\mathrm{CH}_{3} \mathrm{OCHO}$ & $8_{1,7}-7_{1,6}$ & 100.48224 & 22.8 & $1.4 \times 10^{-5}$ & IRAM-30 m & $7.5 \pm 1.9$ & 9.66 & 0.36 \\
\hline $\mathrm{A}-\mathrm{CH}_{3} \mathrm{OCHO}$ & $8_{1,7}-7_{1,6}$ & 100.49068 & 22.8 & $1.4 \times 10^{-5}$ & IRAM-30 m & $5.4 \pm 1.6$ & 9.60 & 0.64 \\
\hline $\mathrm{E}-\mathrm{CH}_{3} \mathrm{OCHO}$ & $9_{0,9}-8_{0,8}$ & 100.68154 & 24.9 & $1.5 \times 10^{-5}$ & IRAM-30 m & $6.3 \pm 1.7$ & 9.54 & 0.70 \\
\hline $\mathrm{A}-\mathrm{CH}_{3} \mathrm{OCHO}$ & $9_{0,9}-8_{0,8}$ & 100.68337 & 24.9 & $1.5 \times 10^{-5}$ & IRAM-30 m & $4.4 \pm 1.5$ & 9.59 & 0.23 \\
\hline $\mathrm{E}-\mathrm{CH}_{3} \mathrm{OCHO}$ & $8_{2,6}-7_{2,5}$ & 103.46657 & 24.6 & $1.5 \times 10^{-5}$ & IRAM-30 m & $<3.1$ & & \\
\hline $\mathrm{A}-\mathrm{CH}_{3} \mathrm{OCHO}$ & $8_{2,6}-7_{2,5}$ & 103.47866 & 24.6 & $1.5 \times 10^{-5}$ & IRAM-30 m & $5.6 \pm 1.6$ & 9.50 & 0.50 \\
\hline
\end{tabular}




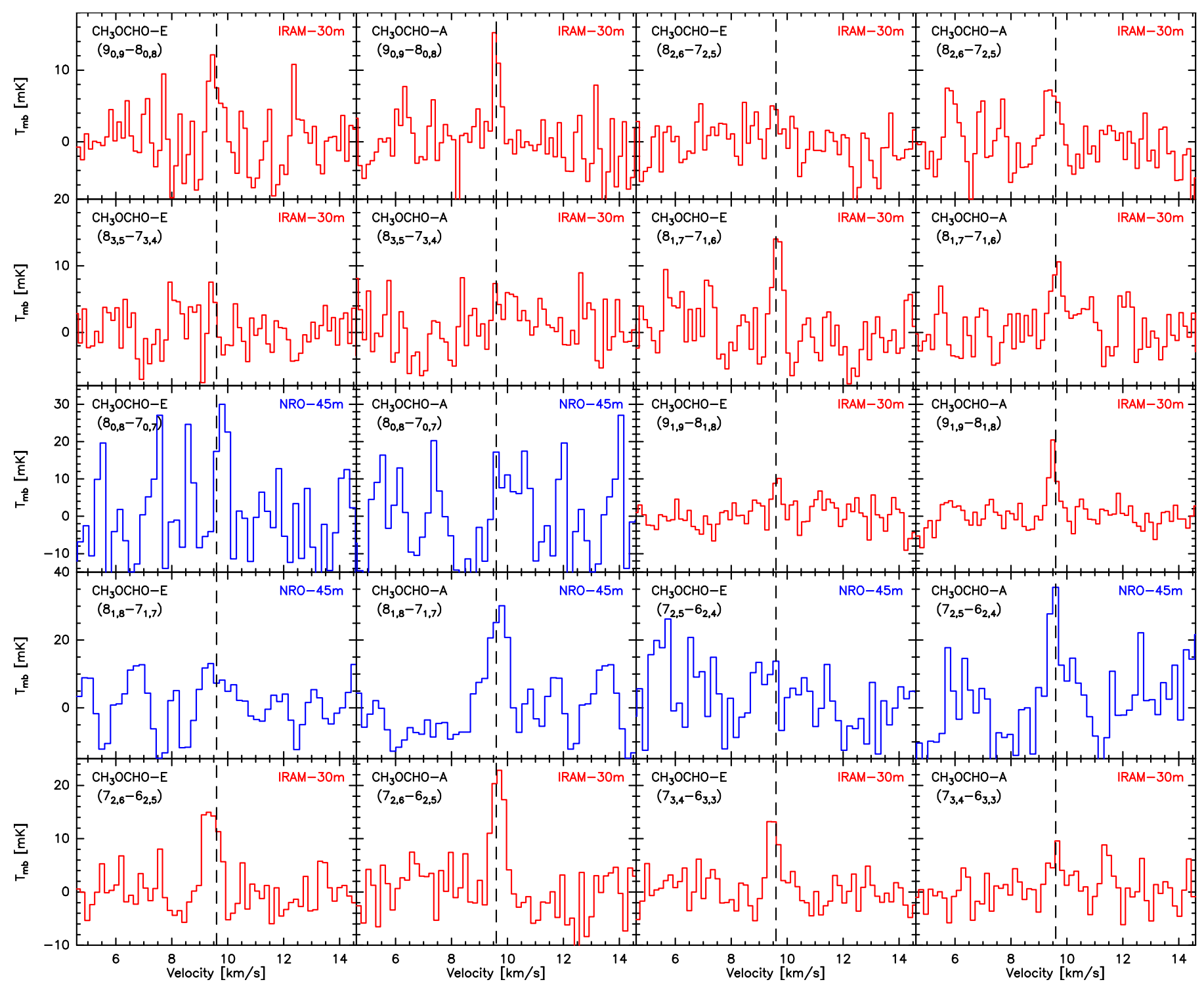

Fig. 8. Spectra of the targeted $\mathrm{CH}_{3} \mathrm{OCHO}$ transitions towards the $\mathrm{B} 5$ methanol hotspot.

the $T_{\text {kin }}-n_{\mathrm{H} 2}, T_{\text {kin }}-N_{\text {tot }}$, and $N_{\text {tot }}-n_{\mathrm{H} 2}$ planes. A finer grid was then performed around the best-fit values with linear steps. Methanol observations can be reproduced with a reduced $\chi^{2}$ of 4.5 for $T_{\text {kin }}=7.5 \pm 1.5 \mathrm{~K}, n_{\mathrm{H} 2}=2.25 \pm 1.50 \times 10^{5} \mathrm{~cm}^{-3}$ and for a methanol column density of $N\left(\mathrm{~A}-\mathrm{CH}_{3} \mathrm{OH}\right)=N\left(\mathrm{E}-\mathrm{CH}_{3} \mathrm{OH}\right)=$ $7.5 \pm 3.0 \times 10^{13} \mathrm{~cm}^{-2}$. The best-fit density is a factor of between two and four higher than the average density in the East 286 dense core, the denser of the two nearby cores (Sadavoy 2013), whilst the kinetic temperature is $2-3 \mathrm{~K}$ and $4-5 \mathrm{~K}$ lower than the average dust temperatures of East 286 and East 189, respectively. This finding would suggest that the methanol emission mostly originates from the centre part of the cores, where the physical conditions are dense and cold. A dedicated analysis of the continuum emission profile of the two dense cores needs to be carried out in order to derive their physical structure. For these physical conditions and this column density, the transitions with integrated brightness temperatures higher than $1 \mathrm{~K} \mathrm{~km} \mathrm{~s}^{-1}$ have an opacity $\tau$ of between one and two showing that some $\mathrm{CH}_{3} \mathrm{OH}$ transitions can be optically thick in B5. The excitation temperatures vary between negative values for the $5_{-1}-4_{0}$, E transition at $84.521 \mathrm{GHz}$ to $\sim 7-7.5 \mathrm{~K}$ for the $\mathrm{E}$ and $\mathrm{A}^{+}$transitions at $\sim 96 \mathrm{GHz}$. In Appendix $\mathrm{A}$ we discuss the impact of physical conditions on the excitation temperatures in more detail.

The narrow range of upper level energies of the methyl formate transitions (between 19 and $25 \mathrm{~K}$ ) prevents us from running a full model grid in which several parameters are varied, since it is impossible to converge towards one single solution that reproduces the observations. Instead, we used the $\mathrm{H}_{2}$ density and the temperature that best reproduce the methanol emission and we only varied the methyl formate column density, first between $10^{11}$ and $10^{15} \mathrm{~cm}^{-2}$ and then on a finer grid between $5 \times 10^{11}$ and $5 \times 10^{12} \mathrm{~cm}^{-2}$. We did not assume any state $\mathrm{E} / \mathrm{A}$ abundance ratio and the $\mathrm{A}-$ and $\mathrm{E}$ - states were treated as two distinct but coexistent species. Methyl formate observations can be reproduced with a reduced $\chi^{2}$ of $\sim 1$ for $N(\mathrm{E}-$ $\left.\mathrm{CH}_{3} \mathrm{OCHO}\right)=2.1 \pm 1.5 \times 10^{12} \mathrm{~cm}^{-2}$ and $N\left(\mathrm{~A}-\mathrm{CH}_{3} \mathrm{OCHO}\right)=$ $2.2 \pm 1.3 \times 10^{12} \mathrm{~cm}^{-2}$. For this density, all transitions are optically thin $(\tau \ll 1)$ and show an excitation temperature $T_{\text {ex }}$ of $6-7 \mathrm{~K}$, close to the kinetic temperature. The HNCO column density that best reproduces the emission of the transition at $87.92525 \mathrm{GHz}$ is $2.0 \pm 0.6 \times 10^{11} \mathrm{~cm}^{-2}$. The results of the RADEX analysis are summarised in Table 3 . 

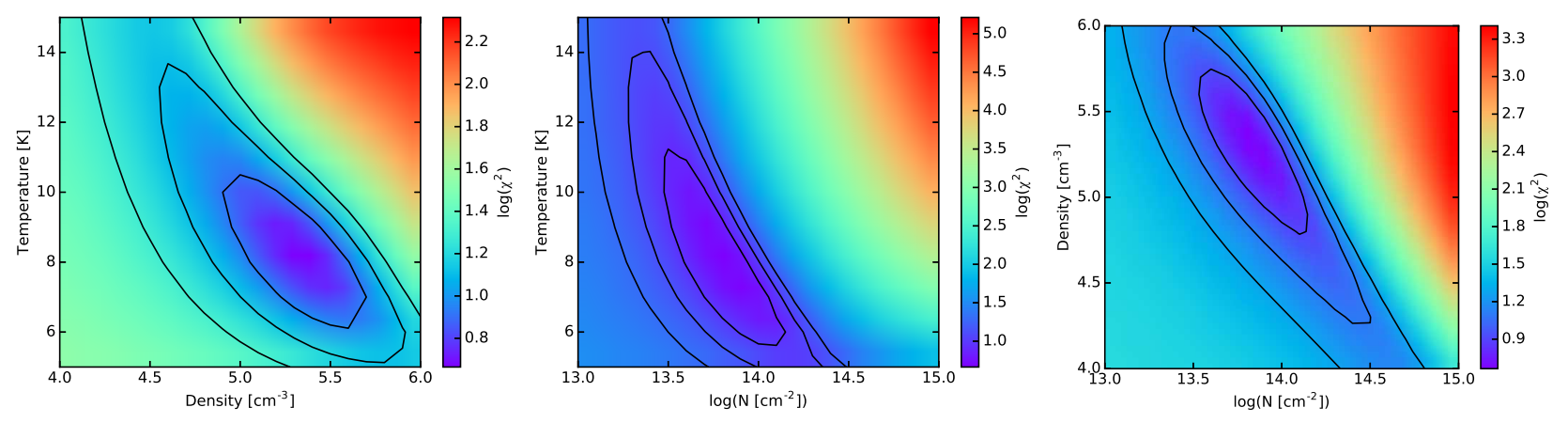

Fig. 9. $\chi^{2}$ distribution in the $\mathrm{H}_{2}$ density - kinetic temperature, column density - kinetic temperature, and column density $-\mathrm{H}_{2}$ density planes for the RADEX fitting of the methanol emission. Black lines show $\chi^{2}$ contours at $1 \sigma, 2 \sigma$, and $3 \sigma$.

Table 3. Results of the RADEX analysis of the methanol and methyl formate emissions.

\begin{tabular}{lccccc}
\hline \hline Molecule & $\begin{array}{c}N_{\text {tot }} \\
\left(\mathrm{cm}^{-2}\right)\end{array}$ & $N_{\text {tot }} / N\left(\mathrm{CH}_{3} \mathrm{OH}\right)$ & $N_{\text {tot }} / N\left(\mathrm{H}_{2}\right)$ & $\begin{array}{c}T_{\text {kin }} \\
(\mathrm{K})\end{array}$ & $\begin{array}{c}n_{\mathrm{H} 2} \\
\left(\mathrm{~cm}^{-3}\right)\end{array}$ \\
\hline $\mathrm{A}-\mathrm{CH}_{3} \mathrm{OH}$ & $7.5(+13) \pm 3.0(+13)$ & & $2.3(-8)$ & $7.5 \pm 1.5$ & $2.25(+5) \pm 1.50(+5)$ \\
$\mathrm{E}-\mathrm{CH}_{3} \mathrm{OH}$ & $7.5(+13) \pm 3.0(+13)$ & & $2.3(-8)$ & $7.5 \pm 1.5$ & $2.25(+5) \pm 1.50(+5)$ \\
$\mathrm{CH}_{3} \mathrm{OH}$ & $1.5(+14) \pm 4.2(+13)$ & & $4.5(-8)$ & $7.5 \pm 1.5$ & $2.25(+5) \pm 1.50(+5)$ \\
\hline $\mathrm{A}-\mathrm{CH}_{3} \mathrm{OCHO}$ & $2.2(+12) \pm 1.3(+12)$ & $1.5(-2)$ & $6.7(-10)$ & $7.5^{a}$ & $2.25(+5)^{a}$ \\
${\mathrm{E}-\mathrm{CH}_{3} \mathrm{OCHO}}_{\mathrm{CH}_{3} \mathrm{OCHO}}^{2.1(+12) \pm 1.5(+12)}$ & $1.4(-2)$ & $6.4(-10)$ & $7.5^{a}$ & $2.25(+5)^{a}$ \\
\hline $\mathrm{HNCO}$ & $2.3(+12) \pm 2.0(+12)$ & $2.9(-2)$ & $1.3(-9)$ & & \\
\hline
\end{tabular}

Notes. ${ }^{(a)}$ The physical conditions are fixed following the results of the methanol analysis.

\subsection{LTE analysis}

The column density of the observed molecules whose collisional coefficients are not known has been obtained through the local thermodynamic equilibrium (LTE) approximation. For the kinetic temperatures $\left(T_{\text {kin }} \sim 8 \mathrm{~K}\right)$ and densities $\left(n_{\mathrm{H}} \sim 10^{5} \mathrm{~cm}^{-3}\right)$ expected towards the methanol hotspot of Barnard 5, the different substates have to be treated separately. The detected acetaldehyde transitions have upper level energies between 5 and $16 \mathrm{~K}$, allowing us to perform a population diagram analysis. The column densities and the rotational temperatures of the two substates are varied to fit the observational data using the routine presented in Taquet et al. (2015) following the Population Diagram method proposed by Goldsmith \& Langer (1999). Table 4 lists the column densities derived at the methanol hotspot of Barnard 5 with the LTE analysis and Fig. 10 presents the Rotational Diagram of acetaldehyde. The rotational temperatures of the two acetaldehyde states are about 5-6 K. This confirms that the observed acetaldehyde transitions are not fully thermalised for such dark cloud conditions.

The transitions of ketene and di-methyl ether have similar upper level energies, preventing us to perform a Rotational Diagram analysis in which the rotational temperature is considered as a free parameter. We therefore only varied the total column density in order to obtain the best fit to the observations assuming two excitation temperatures: $7.5 \mathrm{~K}$, which corresponds to the kinetic temperature in the methanol hotspot derived with the RADEX analysis of the methanol emission, and a lower excitation temperature of $5.0 \mathrm{~K}$ close to the methanol and methyl formate excitation temperatures, and of the acetaldehyde rotational temperature, as should be expected for molecular cloud conditions where levels are usually sub-thermally excited. The four different substates of di-methyl ether were treated

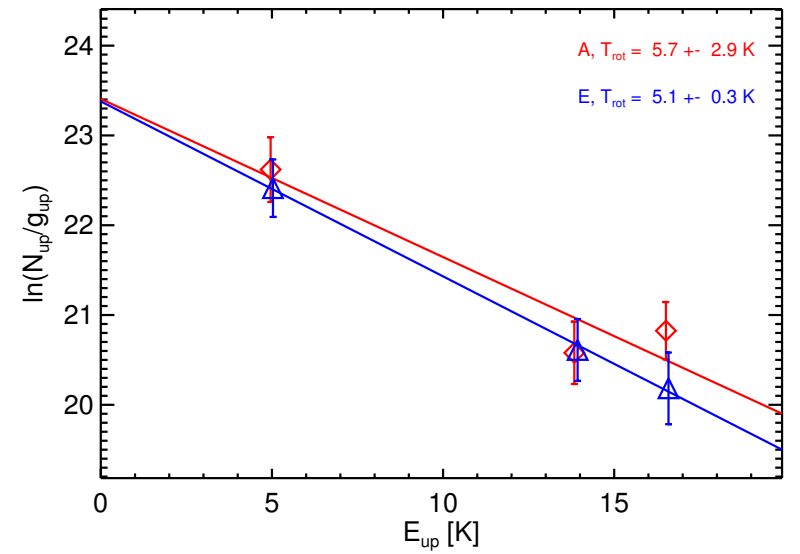

Fig. 10. Rotational diagram of acetaldehyde $\mathrm{CH}_{3} \mathrm{CHO}$. A- and E-states are depicted by red diamonds and blue triangles, respectively.

separately. The EA- and AE- transitions have the same frequency at $99.32443 \mathrm{GHz}$, we therefore considered that the contribution of the two states to the detected transition scales with the degeneracy of their upper energy levels.

As expected, the $\mathrm{A} / \mathrm{E}$ abundance ratio is close to 1 for methyl formate and acetaldehyde. The ortho/para ratio of ketene is equal to $3.3 \pm 1.2$, which is consistent with the statistical ratio of 3.0 and the value of 3.3 and 3.5 found in L1689B and TMC1 (Bacmann et al. 2012; Ohishi et al. 1991).

We also derived the upper limits of other undetected COMs showing at least one bright (i.e., low $E_{\mathrm{up}}$, high $A_{\mathrm{ij}}$ ) transition lying in the observed frequency bands. In particular, glycol aldehyde $\mathrm{HCOCH}_{2} \mathrm{OH}$, an isomer of methyl formate, and ethanol, 
the ethyl counterpart of methanol, show abundance upper limits that are about ten times lower than the species detected in this work.

\section{Discussion}

\subsection{Comparison with other sources}

The methanol hotspot in B5 is located just between two dense cores and therefore represents a stage between the dark cloud and dense core phases. As a consequence, abundances of COMs with respect to methanol, their likely parent species, derived towards B5 listed in Table 4 are compared in Fig. 11 with observations towards the dark clouds TMC1 and L134N and the dense cores B1-b, L1689B, and L1544 (Ohishi et al. 1992; Öberg et al. 2010; Cernicharo et al. 2012; Bacmann et al. 2012; Bizzocchi et al. 2014; Vastel et al. 2014; Bacmann \& Faure 2016; Gratier et al. 2016; Jiménez-Serra et al. 2016). Abundances derived at the methanol hotspot of B5 seem to be in good agreement with observations towards other cold dense cores. In B5, B1-b, and L1689B, all the detected COMs have similar abundances within each source, at least considering the ranges of possible abundances depending on their assumed excitation temperature. However, COMs in B1-b are found to be less abundant with respect to $\mathrm{CH}_{3} \mathrm{OH}$ than in $\mathrm{L} 1689 \mathrm{~B}$ by approximately one order of magnitude. L1544 is the only source showing significant abundance differences between COMs: ketene is ten times more abundant than formic acid and two to four times more abundant than acetaldehyde.

Figure 11 also shows abundance ratios of COMs derived in other types of sources. The Horsehead Nebula PhotonDominated Region (HN PDR) observed by Guzman et al. (2014) is located in the external layer of the Horsehead nebula and undergoes efficient UV photochemistry. It therefore represents the translucent phase prior the formation of dark clouds. Hot cores represent the early stages of star formation when the central source is still embedded in an thick protostellar envelope. Abundances in low-mass protostars are the values derived towards NGC 1333-IRAS2A and IRAS 16293-2422 through sub$\mathrm{mm}$ interferometric observations by Taquet et al. (2015) and Jørgensen et al. (2016), whilst abundances in high-mass protostars are averaged values derived from the compilation by Taquet et al. (2015). Abundances in the comet Hale-Bopp by Bockelee-Morvan et al. (2000) are representative of abundances at the end of the protoplanetary disk phase. Although many other phases are involved in the star formation process, such a comparison can give us a first idea of the abundance evolution of different types of COMs with the evolutionary stage of star formation. The five COMs studied in this work can be distinguished into two categories according to their rotational temperature around massive hot cores (see Isokoski et al. 2013): the lukewarm COMs ketene, acetaldehyde, and formic acid showing low rotational temperatures between 20 and $80 \mathrm{~K}$; and the warm COMs methyl formate and di-methyl ether showing rotational temperatures usually higher than $100 \mathrm{~K}$.

The three lukewarm COMs are found to be as abundant as methanol in the Horsehead PDR but they show abundances of $10-30 \%$ in dark clouds and abundances mostly lower than $10 \%$ in cold dense cores. Around low- and high-mass hot cores, the warm COMs methyl formate and di-methyl ether are more abundant than the three lukewarm COMs. The abundance of $\mathrm{CH}_{2} \mathrm{CO}$ and $\mathrm{CH}_{3} \mathrm{CHO}$ derived in the hot corino of NGC 1333IRAS2A and IRAS 16293-2422 by Taquet et al. (2015) and Jørgensen et al. (2016) are lower than $0.5 \%$ whereas abundances of $\mathrm{CH}_{3} \mathrm{OCHO}$ and $\mathrm{CH}_{3} \mathrm{OCH}_{3}$ are higher than $1 \%$. Abundances of lukewarm COMs around massive hot cores derived from single-dish observations and assuming similar source sizes for all molecules are about $1 \%$ with respect to methanol, and could be even lower if a larger source size is assumed as expected from their low excitation temperature, whilst $\mathrm{CH}_{3} \mathrm{OCHO}$ and $\mathrm{CH}_{3} \mathrm{OCH}_{3}$ abundances are higher than $10 \%$.

\subsection{Ice evaporation processes in dark clouds}

Methanol cannot form in the gas phase (Luca et al. 2002; Garrod \& Herbst 2006) whilst water is the main component of interstellar ices (see Öberg et al. 2011). The detection of cold methanol and water with high abundances of $4.5 \times 10^{-8}$, derived with our RADEX analysis, and $\sim 2 \times 10^{-8}$ w.r.t. $\mathrm{H}_{2}$ (Wirstrom et al. 2014) respectively, in a dark cloud region with no associated infrared emission is therefore attributed to cold grain surface chemistry followed by non-thermal evaporation processes. Several processes have been proposed to trigger evaporation of icy material in the gas phase of dark clouds: 1) photoevaporation induced by the external interstellar and cosmic ray induced field of UV photons; 2) chemical desorption upon surface formation of the molecule; 3 ) ice sputtering by cosmic-rays. A viable desorption mechanism must also account for the spatial chemical differentiations and anti-correlations observed for the different molecules detected in Barnard 5. Processes that are not able to explain the observed spatial distributions can therefore be discounted.

UV photolysis of methanol ice has been experimentally studied by Bertin et al. (2016) and Cruz-Diaz et al. (2016). They showed that UV photolysis of methanol ice mostly results in methanol photodissociation. Bertin et al. (2016) derived a low photoevaporation rate of approximately $10^{-5}$ molecules per incident photon for a pure methanol ice, two orders of magnitude lower than the rates commonly used in astrochemical models. The photoevaporation is found to be an indirect process in which the radicals produced by photodissocation recombine together to release the produced methanol molecule into the gas phase through chemical desorption. UV photolysis of a more realistic methanol-CO ice mixture decreases the rate to $<3 \times 10^{-6}$ molecules per incident photon. The location of the methanol hotspot between two dense cores that can act as shields against external UV irradiation, in combination with its low efficiency, suggests that UV photoevaporation is not the dominant mechanism responsible for its high gas-phase methanol abundance.

The so-called chemical desorption, desorption of a product due to the energy release of the exothermic surface reaction, has been experimentally quantified by Minissale et al. (2016) for a dozen of reactions for an amorphous water ice substrate, representative of ices observed in dense clouds. Although these authors have only been able to derive an upper limit of $8 \%$ for the desorption efficiency of methanol due to the reaction between $\mathrm{CH}_{3} \mathrm{O}$ and $\mathrm{H}$, their theoretical estimate results in a chemical desorption efficiency of $\sim 2 \%$. A high abundance of gaseous methanol between the two dense cores East 189 and East 286 could therefore reflect an efficient methanol formation at the surface of interstellar ices triggering chemical desorption of methanol into the gas phase.

The sputtering efficiency induced by the impact of energetic ions on cold water ice has been studied experimentally by various authors (see Brown et al. 1984). Recently, Dartois et al. (2015) measured the yield of water ice sputtering by swift heavy $\mathrm{MeV}$ ions and derived the sputtering rate of 
Table 4. Column densities and abundances of targetted molecules assuming LTE conditions.

\begin{tabular}{|c|c|c|c|c|c|c|}
\hline \multirow{2}{*}{$\begin{array}{l}\text { Fixed } T_{\text {rot }} \\
\text { Molecule }\end{array}$} & \multicolumn{3}{|c|}{$T_{\text {rot }}=8 \mathrm{~K}$} & \multicolumn{3}{|c|}{$T_{\text {rot }}=5 \mathrm{~K}$} \\
\hline & $\begin{array}{c}N_{\text {tot }} \\
\left(\mathrm{cm}^{-2}\right)\end{array}$ & $N_{\text {tot }} / N\left(\mathrm{CH}_{3} \mathrm{OH}\right)^{a}$ & $N_{\text {tot }} / N\left(\mathrm{H}_{2}\right)^{b}$ & $\begin{array}{c}N_{\text {tot }} \\
\left(\mathrm{cm}^{-2}\right)\end{array}$ & $N_{\text {tot }} / N\left(\mathrm{CH}_{3} \mathrm{OH}\right)^{a}$ & $N_{\text {tot }} / N\left(\mathrm{H}_{2}\right)^{b}$ \\
\hline trans-HCOOH & $2.6(+12) \pm 6.6(+11)$ & $1.7(-2)$ & $7.9(-10)$ & $7.5(+12) \pm 1.9(+12)$ & $5.0(-2)$ & $2.3(-9)$ \\
\hline cis-HCOOH & $1.7(+11) \pm 4.6(+10)$ & $1.1(-3)$ & $5.2(-11)$ & $4.7(+11) \pm 1.3(+11)$ & $3.1(-3)$ & $1.4(-10)$ \\
\hline $\mathrm{HCOOH}$ & $2.8(+12) \pm 6.6(+11)$ & $1.8(-2)$ & $8.4(-10)$ & $8.0(+12) \pm 1.9(+12)$ & $5.3(-2)$ & $2.4(-9)$ \\
\hline $\mathrm{o}-\mathrm{CH}_{2} \mathrm{CO}$ & $2.1(+12) \pm 4.2(+11)$ & $1.4(-2)$ & $6.4(-10)$ & $4.8(+12) \pm 9.6(+11)$ & $3.2(-2)$ & $1.5(-9)$ \\
\hline $\mathrm{p}-\mathrm{CH}_{2} \mathrm{CO}$ & $6.2(+11) \pm 1.8(+11)$ & $4.1(-3)$ & $1.9(-10)$ & $1.5(+12) \pm 4.3(+11)$ & $1.0(-2)$ & $4.5(-10)$ \\
\hline $\mathrm{CH}_{2} \mathrm{CO}$ & $2.7(+12) \pm 4.6(+11)$ & $1.8(-2)$ & $8.2(-10)$ & $6.3(+12) \pm 1.1(+12)$ & $4.2(-2)$ & $1.9(-9)$ \\
\hline $\mathrm{AA}-\mathrm{CH}_{3} \mathrm{OCH}_{3}$ & $3.1(+11) \pm 1.5(+11)$ & $2.1(-3)$ & $9.4(-11)$ & $4.2(+11) \pm 2.0(+11)$ & $2.8(-3)$ & $1.3(-10)$ \\
\hline $\mathrm{EE}-\mathrm{CH}_{3} \mathrm{OCH}_{3}$ & $1.3(+12) \pm 4.4(+11)$ & $8.7(-3)$ & $3.9(-10)$ & $1.7(+12) \pm 6.1(+11)$ & $1.1(-2)$ & $5.2(-10)$ \\
\hline $\mathrm{AE}-\mathrm{CH}_{3} \mathrm{OCH}_{3}$ & $2.2(+11) \pm 2.3(+11)$ & $1.5(-3)$ & $6.7(-11)$ & $2.9(+11) \pm 1.6(+11)$ & $1.9(-3)$ & $8.8(-11)$ \\
\hline $\mathrm{EA}-\mathrm{CH}_{3} \mathrm{OCH}_{3}$ & $2.2(+11) \pm 2.3(+11)$ & $1.5(-3)$ & $6.7(-11)$ & $2.9(+11) \pm 1.6(+11)$ & $1.9(-3)$ & $8.8(-11)$ \\
\hline $\mathrm{CH}_{3} \mathrm{OCH}_{3}$ & $2.1(+12) \pm 5.7(+11)$ & $1.4(-2)$ & $6.2(-10)$ & $2.7(+12) \pm 6.8(+11)$ & $1.8(-2)$ & $8.2(-10)$ \\
\hline $\mathrm{CH}_{3} \mathrm{CCH}$ & $<7.3(+11)$ & $4.9(-3)$ & $2.2(-10)$ & $<7.3(+12)$ & $4.9(-2)$ & $2.2(-9)$ \\
\hline $\mathrm{HCOCH}_{2} \mathrm{OH}$ & $<2.7(+11)$ & $1.8(-3)$ & $8.3(-11)$ & $<7.3(+11)$ & $4.9(-3)$ & $2.2(-10)$ \\
\hline $\mathrm{aGg}-\left(\mathrm{CH}_{2} \mathrm{OH}\right)_{2}$ & $<4.9(+11)$ & $3.2(-3)$ & $1.5(-10)$ & $<2.3(+12)$ & $1.5(-2)$ & $7.0(-10)$ \\
\hline $\mathrm{gGg}-\left(\mathrm{CH}_{2} \mathrm{OH}\right)_{2}$ & $<1.2(+12)$ & $8.2(-3)$ & $3.7(-10)$ & $<3.5(+12)$ & $2.4(-2)$ & $1.1(-9)$ \\
\hline $\mathrm{C}_{2} \mathrm{H}_{5} \mathrm{OH}$ & $<4.1(+11)$ & $2.8(-3)$ & $1.3(-10)$ & $<1.0(+12)$ & $6.7(-3)$ & $3.1(-10)$ \\
\hline $\mathrm{NH}_{2} \mathrm{CHO}$ & $<2.0(+10)$ & $1.3(-4)$ & $6.1(-12)$ & $<5.4(+10)$ & $3.6(-4)$ & $1.6 \mathrm{E}(-11)$ \\
\hline \multirow[t]{2}{*}{ Rotational diagram } & \multicolumn{6}{|c|}{ Free $T_{\text {rot }}$} \\
\hline & $\begin{array}{c}N_{\mathrm{tot}} \\
\left(\mathrm{cm}^{-2}\right)\end{array}$ & $N_{\text {tot }} / N\left(\mathrm{CH}_{3} \mathrm{OH}\right)^{a}$ & $N_{\text {tot }} / N\left(\mathrm{H}_{2}\right)^{b}$ & $\begin{array}{l}T_{\text {rot }} \\
(\mathrm{K})\end{array}$ & & \\
\hline $\mathrm{A}-\mathrm{CH}_{3} \mathrm{CHO}$ & $2.7(+12) \pm 5.5(+11)$ & $1.8(-2)$ & $8.2(-10)$ & $5.7 \pm 2.9$ & & \\
\hline $\mathrm{E}-\mathrm{CH}_{3} \mathrm{CHO}$ & $2.5(+12) \pm 4.6(+11)$ & $1.7(-2)$ & $7.6(-10)$ & $5.1 \pm 0.3$ & & \\
\hline $\mathrm{CH}_{3} \mathrm{CHO}$ & $5.2(+12) \pm 7.2(+11)$ & $3.5(-2)$ & $1.6(-9)$ & & & \\
\hline
\end{tabular}

Notes. ${ }^{(a)}$ The abundance ratio relative to $\mathrm{CH}_{3} \mathrm{OH}$ is computed with the $\mathrm{CH}_{3} \mathrm{OH}$ column density derived from the $\mathrm{RADEX}$ analysis $\left(N\left(\mathrm{CH}_{3} \mathrm{OH}\right)=\right.$ $1.2 \times 10^{14} \mathrm{~cm}^{-2}$; see text). ${ }^{(b)}$ The $\mathrm{H}_{2}$ column density is equal to $3.3 \times 10^{21} \mathrm{~cm}^{-2}$ (Wirstrom et al. 2014).

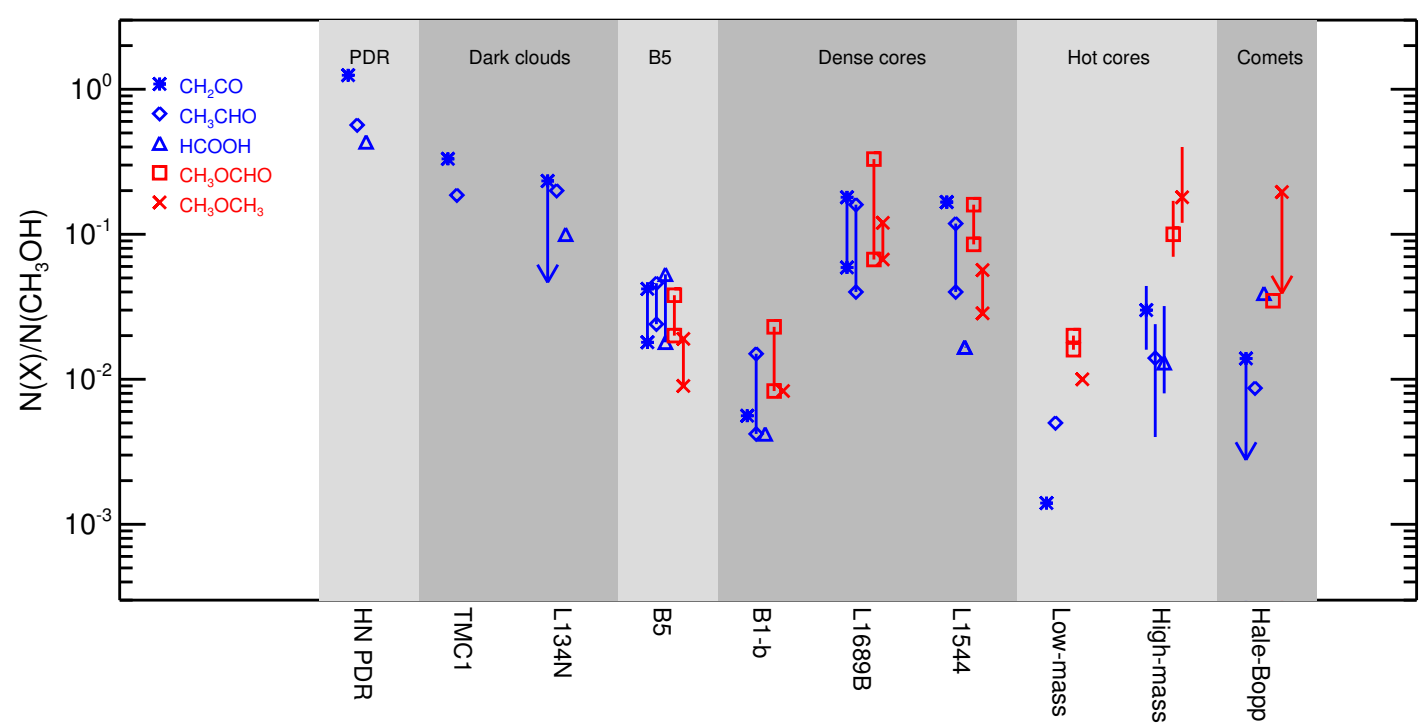

Fig. 11. Abundances of lukewarm (blue) and warm (red) COMs with respect to methanol from PDR regions to comets. Abundances in the Horsehead Nebula Photo-Dissociated Region (HN PDR) are taken from Guzman et al. (2014). Abundances in the TMC1 and L134N dark clouds are from Ohishi et al. (1992) and Gratier et al. (2016). Abundances in the B1-b, L1689B, and L1544 dense cores are taken from Öberg et al. (2010), Cernicharo et al. (2012), Bacmann et al. (2012), Bacmann \& Faure (2016), and Vastel et al. (2014), Bizzocchi et al. (2014), Jiménez-Serra et al. (2016). Abundances in low-mass protostars are those derived in NGC 1333-IRAS2A by Taquet et al. (2015) for $\mathrm{CH}_{2} \mathrm{CO}, \mathrm{CH}_{3} \mathrm{OCHO}$, and $\mathrm{CH}_{3} \mathrm{OCH}_{3}$ and in IRAS 16293-2422-A by Jørgensen et al. (2016) for $\mathrm{CH}_{3} \mathrm{CHO}$, and $\mathrm{CH}_{3} \mathrm{OCHO}$. Abundances in high-mass hot cores are the mean values and the standard deviations of abundances derived from about 20 high-mass hot cores and compiled in Taquet et al. (2015). Abundances in the comet Hale-Bopp are those derived by Bockelee-Morvan et al. (2000). 
water ice for different cosmic ray ionisation rates, which was found to vary between four and 30 molecules $\mathrm{cm}^{-2} \mathrm{~s}^{-1}$. Assuming that the cosmic-ray induced FUV field is about $10^{4}$ photons $\mathrm{cm}^{-2} \mathrm{~s}^{-1}$ (Shen et al. 2004), UV photodesorption would contribute equally to the cosmic ray sputtering with a photodesorption probability of $10^{-3}$. Assuming that methanol has a similar sputtering efficiency than water, this result suggests that ice sputtering by cosmic rays is a much more efficient process than UV photodesorption, by a factor of 100 , for releasing the ice content in the gas phase. The abundance of gas phase methanol induced by cosmic ray ice sputtering would therefore only depend on the total methanol abundance in ices, controlling the total evaporation rate, and the density of the medium, governing the accretion rate. As for chemical desorption, the bright methanol emission seen between two dense cores, and not at the edge of the cores more illuminated by external UV photons, suggests that methanol ice would form efficiently in UV-shielded and colder regions. This result is consistent with the observations of methanol towards the L1544 prestellar core by Bizzocchi et al. (2014) and Spezzano et al. (2016). The methanol emission in L1544 peaks at the northern part of the core where the interstellar radiation field is likely weaker.

As mentioned by Wirstrom et al. (2014), other processes, such as collisions between small gas clumps triggering the heating of interstellar grains due to grain-grain collisions, might also be at work. A detailed analysis of the $\mathrm{CH}_{3} \mathrm{OH}$ maps taken with the IRAM $30 \mathrm{~m}$ will help us to clarify this issue.

\subsection{Detection of cis- $\mathrm{HCOOH}$}

cis-HCOOH has been detected in Barnard 5 at a relative abundance to trans-HCOOH of about $6 \%$, but only from the detection of one single transition at a signal-to-noise of 5 . The detected $4_{0,4}-3_{0,3}$ transition is the cis- $\mathrm{HCOOH}$ line that is expected to be strongest in the $3 \mathrm{~mm}$ band under dark cloud conditions. Assuming a population distribution thermalised at $\sim 8-$ $10 \mathrm{~K}$, only one transition, the $5_{0,5}-4_{0,4}$ line at $109.47 \mathrm{GHz}$, should be detectable at similar noise levels, and while our observations do not cover this frequency, the detection can thus be relatively easily confirmed or refuted. $\mathrm{HCOOH}$ has been shown to form from hydrogenation of $\mathrm{HO}-\mathrm{CO}$ radicals in interstellar ices at low temperatures (Goumans et al. 2008; Ioppolo et al. 2011). The cis-HCOOH isomer lies $1365 \mathrm{~cm}^{-1}$ higher in energy than trans-HCOOH (Lattanzi et al. 2008), and is therefore expected to be about 800 times less abundant around $300 \mathrm{~K}$, and even rarer at the low temperatures of dark clouds. However, a solid state formation mechanism does not necessarily produce an equilibrium isomeric distribution, since the hydrogenation outcome also depends on how the HO-CO complex binds to the surface and its orientation against the incoming $\mathrm{H}$ atom. In addition, the association of $\mathrm{OH}$ and $\mathrm{CO}$ on a grain surface forms $\mathrm{HO}-\mathrm{CO}$ in a trans configuration, which can only isomerise to the slightly higher energy cis-HOCO by overcoming an energy barrier (Goumans et al. 2008). Furthermore, the relative orientation of the $\mathrm{OH}$ group in the cis/trans isomers of $\mathrm{HOCO}$ and $\mathrm{HCOOH}$ give that

cis $-\mathrm{HOCO}+\mathrm{H} \rightarrow$ trans $-\mathrm{HCOOH}$

trans $-\mathrm{HOCO}+\mathrm{H} \rightarrow$ cis $-\mathrm{HCOOH}$.

Thus, it seems possible that a non-negligible fraction of the formic acid formed on grains should be in the cis-configuration.

Cuadrado et al. (2016) recently detected several transitions from cis- $\mathrm{HCOOH}$ towards the Orion Bar photodissociation region and derived a higher cis-to-trans abundance ratio of $36 \%$. This higher ratio could be due to a photoswitching mechanism proposed by these authors in which the absorption of a UV photon by the trans $\mathrm{HCOOH}$ conformer radiatively excites the molecule to overcome the trans-to-cis interconversion barrier. As discussed previously, the methanol hotspot of B5 is likely shielded from strong sources of UV irradiation. Unlike that of the Orion Bar, this photoswitching process is therefore likely inefficient in this dark cloud region.

\subsection{Implication for the formation and the evolution of COMs}

The different trends for the abundance ratio evolution with the evolutionary stage of star formation are consistent with the rotational temperatures derived in high-mass hot cores (see Isokoski et al. 2013). The lukewarm COMs acetaldehyde, ketene, and formic acid should indeed be mostly formed in cold regions either through gas phase chemistry and/or at the surface of interstellar grains. The difference of abundances between PDRs and dark clouds suggests that either the three lukewarm COMs are more efficiently formed with respect to methanol in transluscent regions than in dark clouds or that methanol is more efficiently destroyed through UV photodissociation than ketene, acetaldehyde, and formic acid. To our knowledge, the UV photodissociation cross sections of the three later species are not known and are usually assumed to be equal to those of methanol. As mentioned earlier, the detection of cis- $\mathrm{HCOOH}$ suggests that formic acid would be mostly formed in the inner part of interstellar ices together with $\mathrm{CO}_{2}$ because both molecules can be formed from the reaction between $\mathrm{CO}+\mathrm{OH}$ through the $\mathrm{HO}$ CO complex (Goumans et al. 2008; Ioppolo et al. 2011) and can then be released into the gas phase via chemical desorption. On the other hand, important gas phase formation pathways have been proposed for ketene and acetaldehyde. Cold ketene is mostly formed from the electronic recombination of $\mathrm{CH}_{3} \mathrm{CO}^{+}$ produced by ion-neutral chemistry whilst cold acetaldehyde would be mostly produced through the neutral-neutral reaction between $\mathrm{C}_{2} \mathrm{H}_{5}$ and $\mathrm{O}$ and via the electronic recombination of $\mathrm{CH}_{3} \mathrm{CHOH}^{+}$produced by ion-neutral reactions such as $\mathrm{CH}_{4}+\mathrm{H}_{2} \mathrm{CO}^{+}$(Occhiogrosso et al. 2014). As mentioned in the introduction, surface formation routes producing these two species have also been proposed either through atomic carbon addition of CO followed by hydrogenation (Charnley 1997) or through radical-radical recombination triggered by the UV photolysis of the main ice components during the warm-up phase (Garrod et al. 2008). The simultaneous detection in high abundances of $\mathrm{CH}_{3} \mathrm{CHO}$ and $\mathrm{HCOOH}$ along with $\mathrm{H}_{2} \mathrm{O}$ and $\mathrm{CH}_{3} \mathrm{OH}$ is strongly suggestive of a surface origin. However, the former surface reaction scheme has not been experimentally confirmed yet, whilst the latter is known to be ineffective under laboratory conditions (Öberg et al. 2009).

As mentioned in the Introduction, a small fraction of methyl formate and di-methy ether could be formed in cold conditions, either through neutral-neutral and ion-neutral reactions in the gas or through radical recombination induced by the $\mathrm{CO}$ hydrogenation in interstellar ices. Cold surface formation of COMs from radical-radical recombination induced by addition and abstraction reactions of $\mathrm{CO}, \mathrm{H}_{2} \mathrm{CO}$, and $\mathrm{CH}_{3} \mathrm{OH}$ has been experimentally demonstrated by Fedoseev et al. (2015) and Chuang et al. (2016). However, these experiments tend to produce more glycolaldehyde $\mathrm{HCOCH}_{2} \mathrm{OH}$ than its isomer methyl formate $\mathrm{CH}_{3} \mathrm{OCHO}$, especially when methanol ice is used as initial substrate, because abstraction reactions of methanol only occur on the methyl group favouring the production of $\mathrm{CH}_{2} \mathrm{OH}$ 
with respect to $\mathrm{CH}_{3} \mathrm{O}$. However, glycolaldehyde was not detected towards $\mathrm{B} 5$ and we derived a $\left[\mathrm{HCOCH}_{2} \mathrm{OH}\right] /\left[\mathrm{CH}_{3} \mathrm{OCHO}\right]$ abundance ratio lower than $6 \%$. Assuming that the evaporation efficiency is similar for the two isomers, the low abundance ratio suggests that radical-radical recombination on ices, followed by evaporation, is not the dominant mechanism for the formation of gas phase COMs in dark clouds. Gas phase chemistry triggered by the evaporation of methanol seems to be efficient enough to produce methyl formate and di-methyl ether in similar quantities with abundances of a few percents with respect to methanol (Balucani et al. 2015; Jiménez-Serra et al. 2016). We note that these results depend strongly on the choice of poorly constrained rate coefficients for some key gas phase reactions.

The higher abundances of these two COMs in protostellar cores with respect to the lukewarm species suggests either that the bulk of their molecular material is formed afterwards under warmer conditions or that the lukewarm COMs are destroyed under warmer conditions. High angular resolution observations, allowing us to spatially resolve the hot cores around high- and low-mass sources, are therefore needed to follow the evolution of these complex species with the evolutionary stage of star formation.

\section{Conclusions}

This work presents the detection of several COMs towards the methanol hotspot located between two dense cores of the Barnard 5 Molecular Cloud. Through the use of LTE and nonLTE methods, we have been able to derive the abundances of the targeted COMs with respect to methanol, their likely parent molecule. We summarise here the main conclusions of this work:

1) The efficient non-thermal evaporation of methanol and water is accompanied by the detection of various COMs in high abundances, between $\sim 1$ and $\sim 10 \%$ with respect to methanol.

2) Formic acid has been detected in its two conformers trans and cis. The cis/trans abundance ratio of $6 \%$ confirms that formic acid would be mostly formed at the surface of interstellar grains and then released into the gas phase through non-thermal evaporation processes since the photoswitching mechanism proposed by Cuadrado et al. (2016) can be ruled out in this region.

3) The non-LTE RADEX analysis of the methanol emission allowed us to constrain precisely the physical conditions associated with the observed emission. The methanol emission would originate from a dense $\left(n_{\mathrm{H} 2} \sim 2 \times 10^{5} \mathrm{~cm}^{-3}\right)$ and cold $\left(T_{\text {kin }}=7-8 \mathrm{~K}\right)$ gas, consistent with the centre part of dense cores, compatible with the findings by Bacmann \& Faure (2016) in a sample of prestellar cores.

4) Our targeted COMs can be defined as lukewarm or warm species, according to their rotational temperatures in a sample of massive hot cores (see Isokoski et al. 2013). Comparison with other observations confirms that lukewarm and warm COMs form and show similar abundances in lowdensity cold gas, just as in dense cores - unlike in protostellar cores where the warm COMs tend to be more abundant than the lukewarm species. The evolution of abundances from dark clouds to protostellar cores suggests either that warm COMs are indeed mostly formed in protostellar environments and/or that lukewarm COMs are efficiently destroyed in warm conditions.

5) The low [glycol aldehyde]/[methyl formate] abundance ratio of $<6 \%$ suggests that surface chemistry is not the dominant mechanism for the formation of methyl formate, and possibly also of di-methyl ether.

Acknowledgements. The authors are grateful to the IRAM staff for successfully carrying out the IRAM $30 \mathrm{~m}$ observations and to the NRO staff for their excellent support. V. T. thanks S. Sadavoy for sharing the properties of the Barnard 5 dense cores and N. Sakai for her help in preparing the NRO observations. Astrochemistry in Leiden is supported by the European Union A-ERC grant 291141 CHEMPLAN. E.S.W. acknowledges generous financial support from the Swedish National Space Board. S.B.C. is supported by NASA's Emerging Worlds and the Goddard Center for Astrobiology.

\section{References}

André, P., Men'shchikov, A., Bontemps, S., et al. 2010, A\&A, 518, L102 Bacmann, A., \& Faure, A. 2016, A\&A, 587, A130

Bacmann, A., Taquet, V., Faure, A., et al. 2012, A\&A, 541, L12

Balucani, N., Ceccarelli, C., \& Taquet, V. 2015, MNRAS, 449, L16

Batrla, W., \& Menten, K. M. 1988, ApJ, 329, L117

Belloche, A., Garrod, R. T., Müller, H. S. P., et al. 2009, A\&A, 499, 215

Belitsky, V., Lapkin, I., Fredrixon, M., et al. 2015, A\&A, 580, A29

Bertin, M., Romanzin, C., Doronin, M., et al. 2016, ApJ, 817, L12

Bisschop, S. E., Jørgensen, J. K., van Dishoeck, E. F., \& de Wachter, E. B. M. 2007, A\&A, 465, 913

Bizzocchi, L., Caselli, P., Spezzano, S., \& Leonardo, E. 2014, A\&A, 569, A27

Blake, G. A., Sutton, E. C., Masson, C. R., \& Phillips, T. G. 1987, ApJ, 315, 621

Bockelée-Morvan, D., Lis, D. C., Wink, J. E., et al. 2000, A\&A, 353, 1101

Bottinelli, S., Ceccarelli, C., Lefloch, B., et al. 2004a, ApJ, 615, 354

Bottinelli, S., Ceccarelli, C., Neri, R., et al. 2004b, ApJ, 617, L69

Bottinelli, S., Ceccarelli, C., Williams, J. P., \& Lefloch, B. 2007, A\&A, 463, 601 Brown, W. L., Augustyniak, W. M., Marcantonio, K. J., et al. 1984, Nucl. Instr. Meth. Phys. Res. B, 1, 307

Caselli, P., Keto, E., Pagani, L., et al. 2010, A\&A, 521, L29

Caselli, P., Keto, E., Bergin, E. A., et al. 2012, ApJ, 759, L37

Cazaux, S., Tielens, A. G. G. M., Ceccarelli, C., et al. 2003, ApJ, 593, L51

Cernicharo, J., Marcelino, N., Roueff, E., et al. 2012, ApJ, 759, L43

Charnley, S. B. 1997, IAU Colloq., 161, 89

Charnley, S. B., Tielens, A. G. G. M., \& Millar, T. J. 1992, ApJ, 399, L71

Chuang, K.-J., Fedoseev, G., Ioppolo, S., van Dishoeck, E. F., \& Linnartz, H. 2016, MNRAS, 455, 1702

Coutens, A., Persson, M. V., Jørgensen, J. K., Wampfler, S. F., \& Lykke, J. M. 2015, A\&A, 576, A5

Crapsi, A., Caselli, P., Walmsley, M. C., \& Tafalla, M. 2007, A\&A, 470, 221

Cruz-Diaz, G. A., Martín-Doménech, R., Muñoz Caro, G. M., \& Chen, Y.-J. 2016, A\&A, 592, A68

Cuadrado, S., Goicoechea, J. R., Roncero, O., et al. 2016, A\&A, 596, L1

Dartois, E., Augé, B., Boduch, P., et al. 2015, A\&A, 576, A125

Faure, A., Remijan, A. J., Szalewicz, K., \& Wiesenfeld, L. 2014, ApJ, 783, 72

Fedoseev, G., Cuppen, H. M., Ioppolo, S., Lamberts, T., \& Linnartz, H. 2015, MNRAS, 448, 1288

Friberg, P., Hjalmarson, A., Madden, S. C., \& Irvine, W. M. 1988, A\&A, 195, 281

Garrod, R. T. 2013, ApJ, 765, 60

Garrod, R. T., \& Herbst, E. 2006, ApJ, 936, 13

Garrod, R. T., Weaver, S. L. W., \& Herbst, E. 2008, ApJ, 682, 283

Geppert, W. D., Hamberg, M., Thomas, R. D., et al. 2006, Faraday Discussions, 133, 177

Gérin, M., Pety, J., Fuente, A., et al. 2015, A\&A, 577, L2

Gibb, E. L., Whittet, D. C. B., Boogert, A. C. A., \& Tielens, A. G. G. M. 2004, ApJS, 151, 35

Goldsmith, P. F., \& Langer, W. D. 1999, ApJ, 517, 209

Goumans, T. P. M., Uppal, M. A., \& Brown, W. A. 2008, MNRAS, 384, 1158

Gratier, P., Majumdar, L., Ohishi, M., et al. 2016, ApJS, 225, 25

Green, S. 1986, NASA Technical Memorandum 87791

Guzmán, V. V., Pety, J., Gratier, P., et al. 2014, Faraday Discussions, 168, 103

Hatchell, J., Richer, J. S., Fuller, G. A., et al. 2005, A\&A, 440, 151

Herbst, E., \& van Dishoeck, E. F. 2009, ARA\&A, 47, 427

Hirota, T., Bushimata, T., Choi, Y. K., Honma, M., et al. 2008, PASJ, 60, 37

Horn, A., Møllendal, H., Sekiguchi, O., et al. 2004, ApJ, 611, 605

Ioppolo, S., Cuppen, H. M., van Dishoeck, E. F., \& Linnartz, H. 2011, MNRAS, 410, 1089

Irvine, W. M., Friberg, P., Kaifu, N., et al. 1989, ApJ, 342, 871

Irvine, W. M., Friberg, P., Kaifu, N., Matthews, H. E., et al. 1990, A\&A, 229, L9

Isokoski, K., Bottinelli, S., \& van Dishoeck, E. F. 2013, A\&A, 554, A100

Jimenénez-Serra, I., Vasyunin, A. I., Caselli, P., et al. 2016, ApJ, 830, L6 
Jørgensen, J. K., Bourke, T. L., Myers, P. C., et al. 2005, ApJ, 632, 973

Jørgensen, J. K., Bourke, T. L., Nguyen Luong, Q., \& Takakuwa, S. 2011, A\&A, 534, A 100

Jørgensen, J. K., Favre, C., Bisschop, S. E., et al. 2012, ApJ, 757, L4

Jørgensen, J. K., van der Wiel, M. H. D., Coutens, A., et al. 2016, A\&A, 595 A117

Kuan, Y.-J., Huang, H.-C., Charnley, S. B., et al. 2004, ApJ, 616, L27

Lattanzi, V., Walters, A., Drouin, B. J., \& Pearson, J. C. 2008, ApJS, 176, 536

Luca, A., Voulot, A. D., \& Gerlich, D. D. 2002, WDS 2002 (Prague) Proc. Contributed Papers, Part II, 294

Matthews, H. E., Friberg, P., \& Irvine, W. M. 1985, ApJ, 290, 609

Maury, A. J., Belloche, A., André, P., et al. 2014, A\&A, 563, L2

Millar, T. J., Herbst, E., \& Charnley, S. B. 1991, ApJ, 369, 147

Minissale, M., Dulieu, F., Cazaux, S., \& Hocuk, S. 2016, A\&A, 585, A24

Öberg, K. I., Garrod, R. T., van Dishoeck, E. F., \& Linnartz, H. 2009, A\&A, 504, 891

Öberg, K. I., Bottinelli, S., Jørgensen, J. K., \& van Dishoeck, E. F. 2010, ApJ, 716,825

Öberg, K. I., Boogert, A. C. A., Pontopiddan, K. M., et al. 2011, ApJ, 740, 109

Occhiogrosso, A., Vasyunin, A., Herbst, E., et al. 2014, A\&A, 564, A123
Ohishi, M., \& Kaifu, N. 1998, Faraday Discussions, 109, 205

Ohishi, M., Kawaguchi, K., Kaifu, N., et al. 1991, ASP Conf. Ser., 16, 387

Ohishi, M., Irvine, W. M., \& Kaifu, N. 1992, IAU Symp., 150, 171

Persson, M. V., Jørgensen, J. K., \& van Dishoeck, E. F. 2012, A\&A, 541, A39

Rabli, D., \& Flower, D. R. 2010, MNRAS, 406, 95

Reboussin, L., Wakelam, V., Guilloteau, S., \& Hersant, F. 2014, MNRAS, 440, 3557

Sadavoy, S. I. 2013, Ph.D. Thesis, University of Victoria

Shen, C. J., Greenberg, J. M., Schutte, W. A., \& van Dishoeck, E. F. 2004, A\&A, 415, 203

Spezzano, S., Bizzocchi, L., Caselli, P., Harju, J., \& Brünken, S. 2016, A\&A, 592, L11

Taquet, V., López-Sepulcre, A., Ceccarelli, C., et al. 2015, ApJ, 804, 81

Taquet, V., Wirström, E., \& Charnley, S. B. 2016, ApJ, 821, 46

Tercero, B., Cernicharo, J., López, A., et al. 2015, A\&A, 582, L1

Van der Tak, F. F. S., Black, J. H., Schöier, F. L., Jansen, D. J., \& van Dishoeck, E. F. 2007, A\&A, 468, 627

Vastel, C., Ceccarelli, C., Lefloch, B., \& Bachiller, R. 2014, ApJ, 795, L2

Vasyunin, A. I., \& Herbst, E. 2013, ApJ, 769, 34

Wirström, E. S., Charnley, S. B., Persson, C. M., et al. 2014, ApJ, 788, L32 


\section{Appendix A: Excitation of methanol and methyl formate levels}

The excitation temperatures computed with RADEX for the observed methanol and methyl formate transitions as function of the $\mathrm{H}_{2}$ density are shown in Figs. A.1 and A.2 respectively. The column density is the best-fit value listed in Table 3 and $T_{\text {kin }}$ is set to $7.5 \mathrm{~K}$. All the methyl formate transitions follow the same trend, their excitation temperatures increase with density from $\sim 3 \mathrm{~K}$ at $10^{3} \mathrm{~cm}^{-3}$ to reach the kinetic temperature at densities higher than $10^{6} \mathrm{~cm}^{-3}$ showing that most methyl formate transitions in the $3 \mathrm{~mm}$ band should be thermalised for densities higher than $10^{6} \mathrm{~cm}^{-3}$. For methanol, the situation is more complex. The E- $\mathrm{CH}_{3} \mathrm{OH}$ transition at $84.521 \mathrm{GHz}$ shows a negative excitation temperature for densities higher than $10^{4} \mathrm{~cm}^{-3}$, showing that this inversion population of the $5_{-1}-4_{0}$ doublet is a robust phenomenon. This transition has been known to be a strong maser since its detection towards the massive protostar DR21(OH) by Batrla \& Menten (1988), explaining its bright emission in our data. Other $\mathrm{CH}_{3} \mathrm{OH}$ transitions show positive excitation temperatures but do not follow the same trend with the density. The two $J_{0}-J_{-1}$, E- transitions at $157 \mathrm{GHz}$ show lower $(\sim 3 \mathrm{~K})$ excitation temperatures than other transitions. Their excitation temperatures remain lower than $5 \mathrm{~K}$ even at high densities whilst the excitation temperature of other transitions is close to the kinetic temperature. As already shown in many previous publications, the thermalisation conditions of the $\mathrm{CH}_{3} \mathrm{OH}$ transitions strongly depend on their critical densities, which can highly vary between different upper level quantum numbers. As an example, the detected $J_{0}-J_{-1}(J=1,2)$, E- transitions have critical densities higher than $10^{6} \mathrm{~cm}^{-3}$ whilst the $2_{K}-1_{K}(K=-1,0)$, E- transitions show critical densities of $2-3 \times 10^{4} \mathrm{~cm}^{-3}$ at $10 \mathrm{~K}$, explaining their different excitation temperatures.

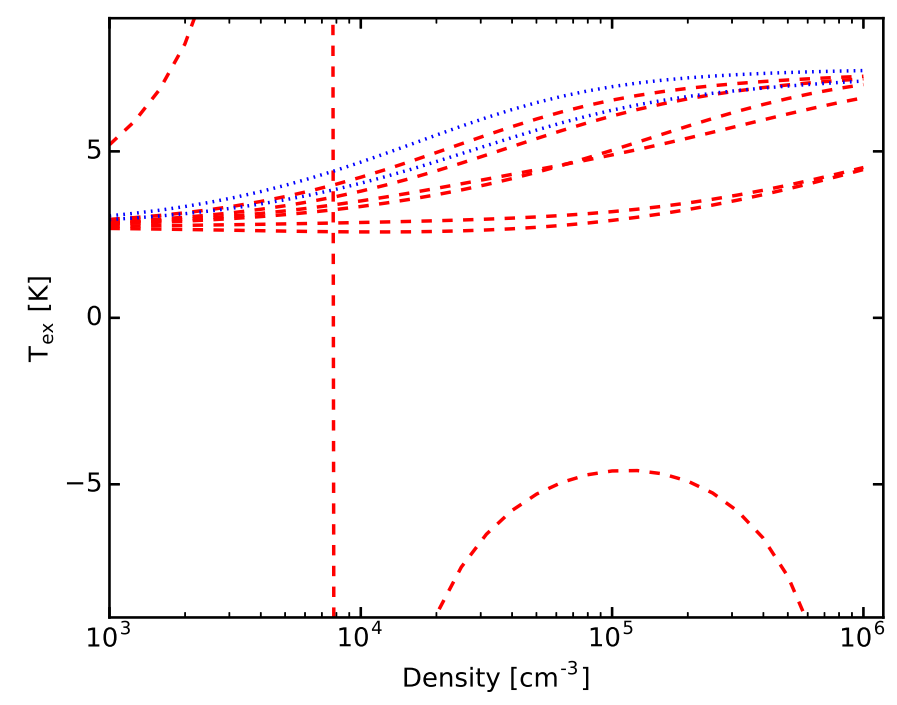

Fig. A.1. Excitation temperatures of the methanol transitions as function of the $\mathrm{H}_{2}$ density for $T_{\text {kin }}=7.5 \mathrm{~K}$. The blue dashed curve shows the A-state transition whilst the red solid curves show the E-state transitions. The transition showing an inversion population is the one at $84.521 \mathrm{GHz}$.

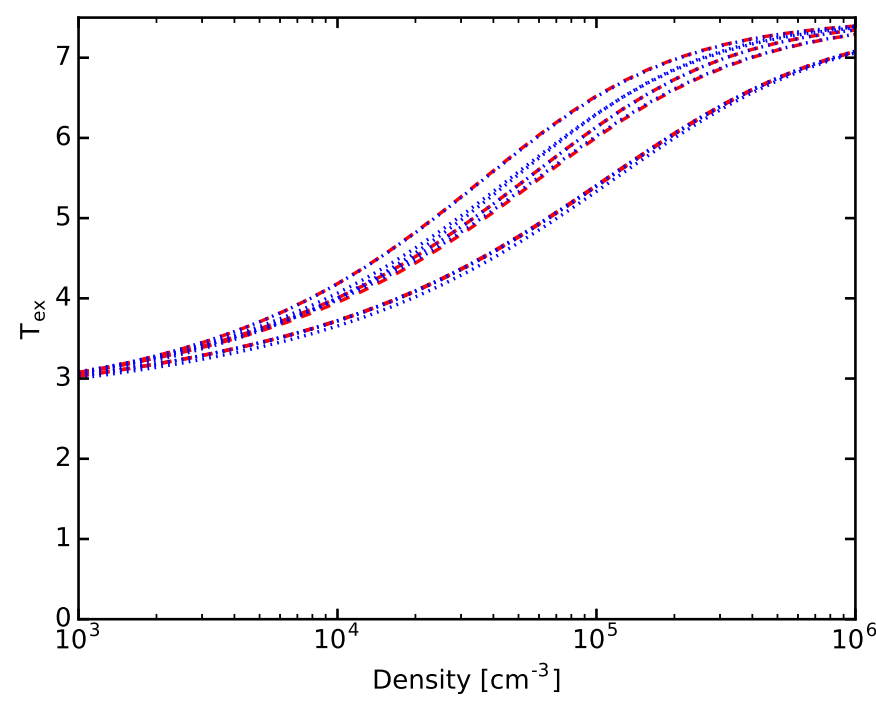

Fig. A.2. Excitation temperatures of the methyl formate transitions as function of the $\mathrm{H}_{2}$ density for $T_{\text {kin }}=7.5 \mathrm{~K}$. The blue dotted curves show the A-state transitions whilst the red dashed curves show the E-state transitions. 